\title{
A prospective audit of the use of diagnostic laparoscopy to establish the diagnosis of abdominal tuberculosis
}

\author{
By \\ J Islam \\ MBBS (Dhaka), DA (SA), FCS (SA)
}

Submitted for the requirement for the degree of

\section{Master of Medical Science in Surgery}

In the

Department of Surgery

Nelson R Mandela School of Medicine

University of Kwa-Zulu Natal

2011 


\title{
Supervisor:
}

\author{
Professor SR Thomson \\ MCh, FRCS (Eng \& Ed) \\ Professor of Surgery, University of Cape Town
}

\section{Co-supervisors:}

DL Clarke

MBBCh, FCS (SA), MMed Sci (Natal), MBA (Natal)

Principal Specialist, Department of Surgery

Grey's Hospital, Pietermaritzburg

\author{
D Wilson \\ MBChB, FCP (SA) \\ Head, Department of Medicine \\ Edendale Hospital, Pietermaritzburg
}

H Dawood

BSc, MBBCh, FCP (SA), LLM

Principal specialist, Department of Medicine

Grey's Hospital, Pietermaritzburg 


\section{Declaration}

I, DR. JAHANGIRUL ISLAM declare that:

(i) The research reported in this dissertation, except where otherwise indicated, is my original work.

(ii) This dissertation has not been submitted for any degree or examination at any other university.

(iii) This dissertation does not contain other person's data, pictures, graphs or other information, unless specifically acknowledged as being sourced from other persons.

(iv) This dissertation does not contain other person's writing, unless specifically acknowledged as being sourced from other researchers. Where other written sources have been quoted, then:

a) their words have been re-written but the general information attributed to them has been referenced;

b) where their exact words have been used, their writing has been placed inside quotation marks, and referenced. 
(v) Where I have reproduced a publication of which I am an author, co-author or editor, I have indicated in detail which part of the publication was actually written by myself alone and have fully referenced such publications.

(vi) This dissertation does not contain text, graphics or tables copied and pasted from the Internet, unless specifically acknowledged, and the source being detailed in the dissertation and in the References sections.

Signed: Date:

Name: Jahangirul Islam

Principal investigator

Signed: Date:

Name: Prof. SR Thomson

Supervisor 


\section{Dedication}

To my loving wife, Ferdousi and wonderful sons, Fahmidul and Navidul.

Without their love, support and sacrifice, this work would not have been possible. 


\section{Acknowledgements}

This work has been only possible with the selfless support of a number of individuals.

Fernando Ghimenton, a surgical mentor who was the inspiration of my surgical career and Victor Govindasamy, a senior colleague who was always very helpful in need. Damian Clarke, a colleague and co-supervisor, who inspired me with the idea of this work and Sandie Thomson, my supervisor who has given me a lot of his valuable time.

I am deeply indebted to all of these people.

Thank you. 


\section{Abstract}

\section{Introduction:}

HIV epidemic is one of the major challenges to the South Africa's socio-economic development. The incidence of tuberculosis is rising in sub-Saharan Africa, and in 2009 South Africa had the second highest incidence of tuberculosis in the world. Approximately $80 \%$ of incident tuberculosis cases in South Africa are HIV positive. In HIV positive individual, abdominal tuberculosis has been reported as the most common form of extra-pulmonary tuberculosis. HIV/AIDS has resulted in a resurgence of abdominal tuberculosis in South Africa. Making the diagnosis of abdominal tuberculosis is still difficult, though the condition is common. The role of laparoscopy in making the diagnosis is undefined.

\section{Method:}

All patients with clinically and radiologically suspected but histologically or microbiologically unconfirmed abdominal tuberculosis were referred to the investigating team and laparoscopy was performed to diagnose abdominal tuberculosis. Histology was performed on tissue biopsy specimens and TB culture on ascitic fluid and peripheral blood specimens. 


\section{Results:}

From January 2008 to June 2010 a total of 190 patients were referred to us. No surgical intervention was taken in 60 patients; all of them were HIV positive. Twenty six of them died (43\%) in the hospital during the evaluation period before the diagnostic laparoscopy, and the rest (57\%) were unfit for anaesthesia. Forty nine patients required emergency laparotomy either for bowel obstruction or peritonitis and $39 \%$ of them died. Eighty one patients underwent diagnostic laparoscopy and $77 \%$ of them were HIV positive, in $16 \%$ the HIV status was unknown. Two percent had clinical ascites. Laparoscopic findings included intraabdominal lymphadenopathy in 56 , minimal ascitic fluid in 46 , intra-abdominal mass in 17, and deposits on bowel wall, peritoneum or omentum in 20 patients. Fifty five patients (68\%) had positive histology for tuberculosis. In 15 patients (19\%) histology revealed non-specific inflammation, no pathology was found in one patient and no specimen was taken from one patient. Eighty percent of peritoneal deposits and $77 \%$ of lymph nodes were positive for tuberculosis, whereas $35 \%$ ascitic fluid culture was positive. In nine patients $(11 \%)$ an alternative diagnosis was found (appendicitis, adenocarcinoma, lymphoma).

\section{Conclusion:}

Laparoscopy was feasible and showed a high yield to establish the diagnosis of abdominal tuberculosis and to provide an alternate diagnosis. Laparoscopy was useful to establish the gross features of abdominal tuberculosis and to provide 
the adequate specimens for examinations. Very poor follow negated the evaluation of the clinical response to anti tuberculosis therapy. 


\section{Table of contents}

Title page

1

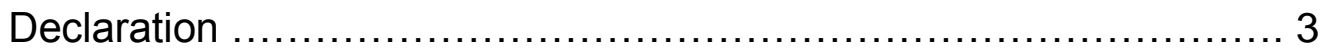

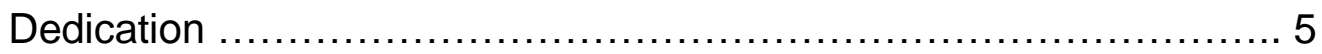

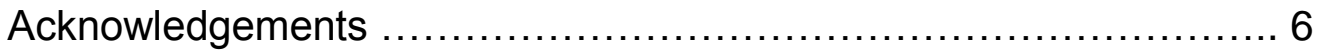

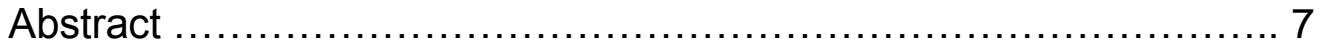

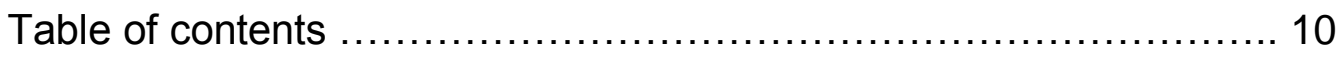

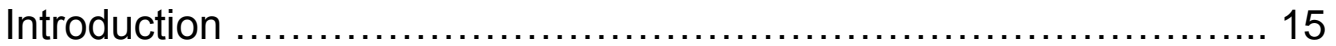

1.1 Human Immunodeficiency Virus disease ................ 15

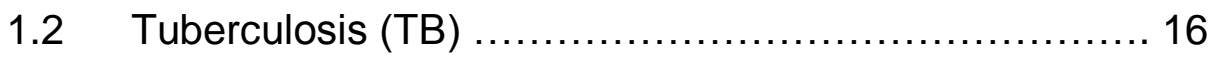

$1.3 \quad \mathrm{HIV} / \mathrm{TB}$ Co-infection ................................. 16

$1.4 \quad$ Abdominal tuberculosis ................................ 18

1.5 Diagnosis of abdominal tuberculosis .................. 20

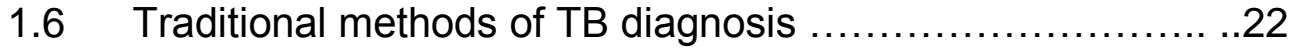

$1.7 \quad$ Alternate diagnostic adjuncts for TB ....................... 22 
$1.8 \quad$ Newer modalities to diagnose TB .......................... 24

1.9 Imaging in the diagnosis of abdominal tuberculosis .......... 26

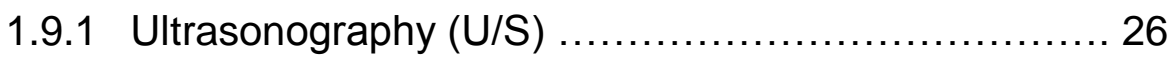

1.9.2 Computerized tomography (CT) scans ............... 26

1.9.3 Upper and lower gastrointestinal series (Barium studies) 27

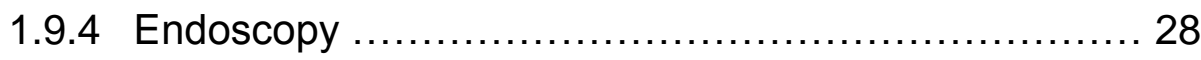

1.10 Laparotomy to diagnose abdominal tuberculosis ............... 28

1.11 History of laparoscopy in diagnosis of abdominal tuberculosis ..30

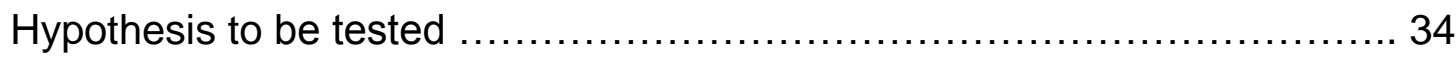

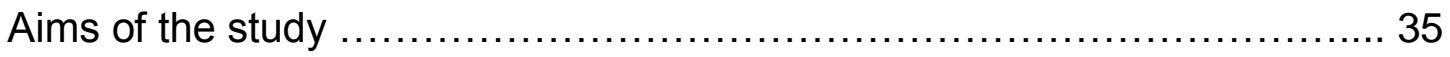

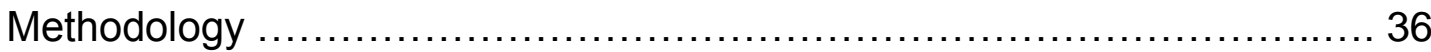

Laparoscopic technique ............................................... 40

Assessing diagnostic accuracy ............................................. 41

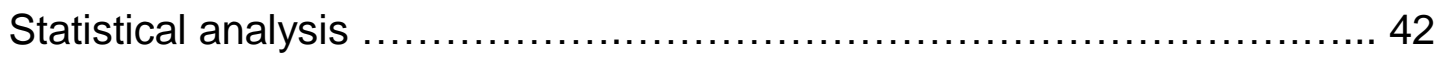

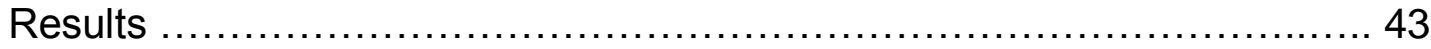

2.1 Group A: Laparoscopy ..................................... 44

2.1.1 Duration of symptoms and hospital stays ................... 44 
2.1.2 Clinical presentation

2.1.3 Preoperative investigations ............................. 46

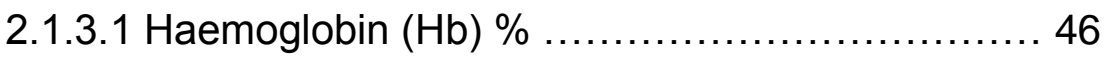

2.1.3.2 White Cells Counts (WCC) .................... 46

2.1.3.3 Neutrophils ................................... 46

2.1.3.4 Lymphocytes ................................. 46

2.1.3.5 Chest X-Ray (CXR) …...................... 47

2.1.3.6 U/S abdomen ................................ 47

2.1.3.7 CT scan abdomen ............................. 47

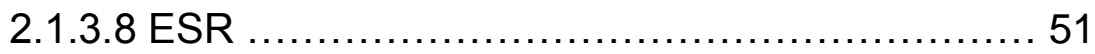

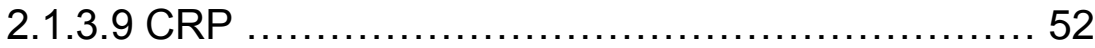

2.1.3.10 Blood culture ................................. 52

2.1.3.11 Endoscopies .................................. 53

2.1.3.12 Contrast studies ............................... 53

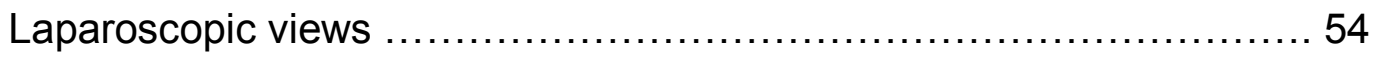

3.1 Lymph nodes .......................................... 54

3.2 Ascitic fluid culture ...................................... 56 
3.4 Tubercles 59

3.5 Peritoneum in the absence of tubercles 60

3.6 Abdominal/ retroperitoneal mass ........................ 61

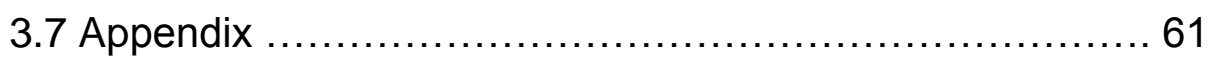

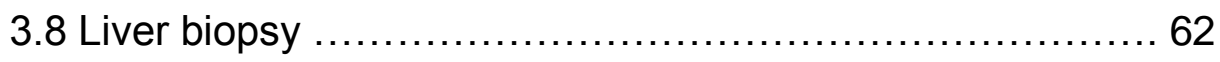

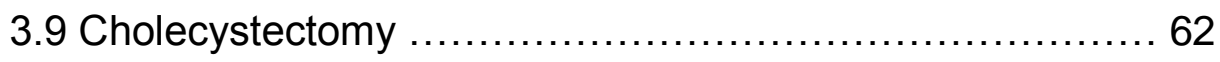

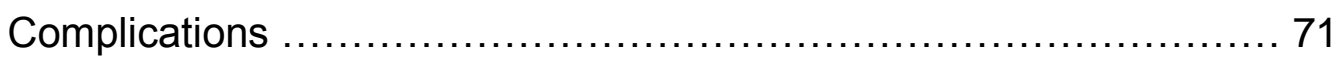

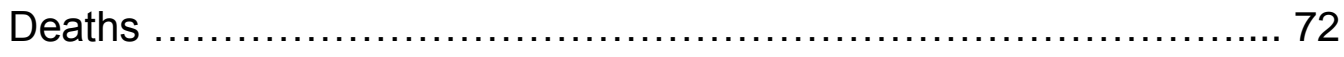

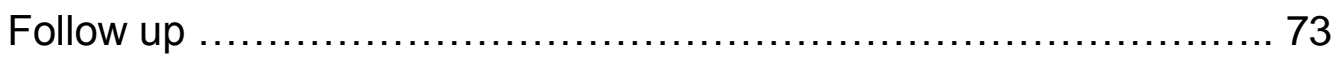

4 Group B: Laparotomy ........................................... 75

5 Group C: No surgical intervention ............................... 76

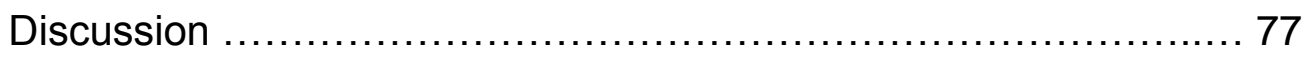

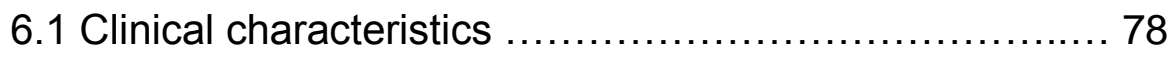

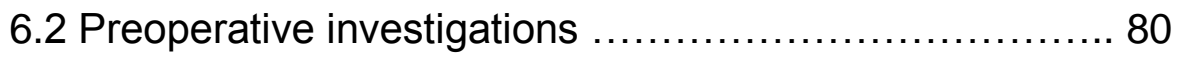

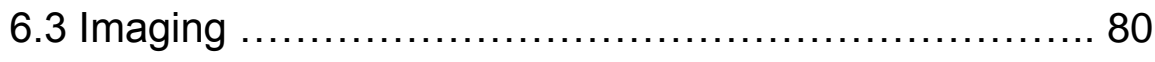

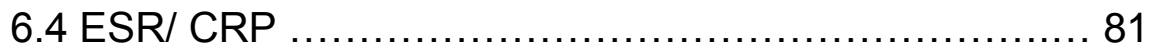


6.5 Blood culture

6.6 PCR

83

6.7 Endoscopy 84

6.8 Laparoscopy ........................................ 84

6.9 Diagnosis other than tuberculosis $\ldots \ldots \ldots \ldots \ldots \ldots \ldots \ldots \ldots \ldots$

6.10 Non-specific chronic inflammations ................. 87

6.11 Perioperative complications/ deaths ................. 88

6.12 Laparotomy and Laparoscopy for TB abdomen ...... 89

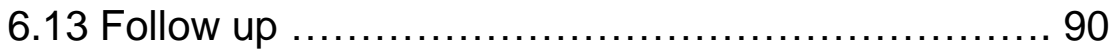

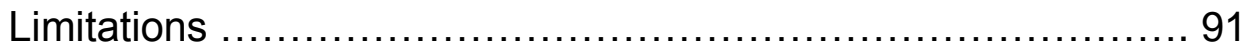

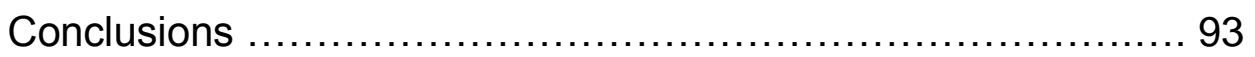

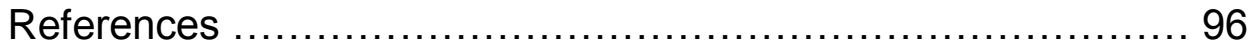

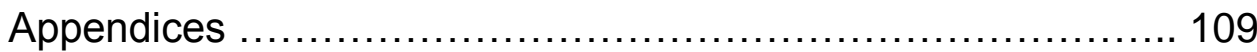

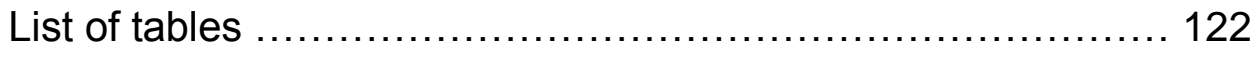

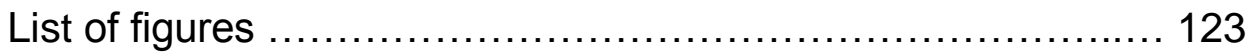

List of abbreviations .............................................. 124 


\section{Introduction}

The current relevant epidemiology of Tuberculosis and Human Immunodeficiency Virus (HIV) and their interactions are outlined seriatim in the following sections. They provide an essential background for the discussion on the diagnosis of abdominal tuberculosis in the HIV era.

\subsection{Human Immunodeficiency Virus disease:}

The HIV epidemic is one of the major challenges to South Africa's socioeconomic development. Worldwide, South Africa has the highest number of people living with HIV/AIDS (Acquired Immune Deficiency Syndrome), representing a quarter of the disease burden in sub-Saharan Africa and a sixth of the global disease burden. ${ }^{1}$ In 2009, an estimated 33.3 million people were living with HIV infection in the world; in South Africa alone it was 5.63 million and 1.8 million people died with AIDS in 2009. ${ }^{2}$ The national HIV prevalence in South Africa in general population for 2009 was $17.8 \%$, which was $4^{\text {th }}$ highest in the world and the highest percentage was in the province of KwaZulu-Natal, which was $25 \% .^{3}$ The highest provincial HIV prevalence among the ante-natal women aged 15-49 years was also recorded in KwaZulu-Natal in 2009 and it was $39.5 \% .^{3}$ 


\subsection{Tuberculosis (TB):}

Globally, there were an estimated 9.4 million new cases of tuberculosis in 2008, the incidence of tuberculosis is increasing by about $0.4 \%$ and approximately two million people die of it annually. ${ }^{4,5}$ The incidence of tuberculosis is rising in subSaharan Africa as well. South Africa had the seventh highest per capita incidence of tuberculosis. In 2003 and in 2009 it had the $2^{\text {nd }}$ highest incidence in the world. ${ }^{6,7}$ In particular, the Western Cape province reported an incidence as high as 1000 cases per 100000 population per annum. ${ }^{8}$ South Africa has one of the worst tuberculosis epidemics in the world, with high disease burden, incidence rates, and HIV co-infection rates, and growing epidemics of multidrugresistant and extensively drug-resistant tuberculosis. ${ }^{1} \mathrm{~A}$ recent report noted that about $28 \%$ of medical admissions in Edendale Hospital, KwaZulu-Natal, were for active tuberculosis and $26.5 \%$ of these patients died in this hospital. ${ }^{4}$ The annual incidence of tuberculosis in KwaZulu-Natal was 1094 cases/100,000 in 2006. ${ }^{9}$ Tuberculosis has become the leading cause of death in South Africa by clinically determined death notification. ${ }^{10}$

\subsection{HIV/ TB Co-infection:}

The increased incidence in tuberculosis in the last decade is related to the HIV pandemic. Approximately $80 \%$ of incident tuberculosis cases in South Africa are HIV positive and these co-infected individuals are more likely to have smearnegative pulmonary or extrapulmonary tuberculosis, which can be very difficult to diagnose clinically. ${ }^{11}$ Deaths from tuberculosis among HIV positive people 
account for $23 \%$ of the estimated two million deaths due to HIV/AIDS in $2007 .{ }^{12}$ Tuberculosis is the most common cause of death in people infected with HIV worldwide, and accounts for $11 \%$ of AIDS deaths. ${ }^{4}$ Tuberculosis and HIV coinfection act synergistically resulting in increased mortality. The incidence of tuberculosis is also increasing in the HIV negative population. HIV infection is a strong risk factor for developing both pulmonary and extrapulmonary tuberculosis. The incidence of extrapulmonary tuberculosis is $10 \%$ to $15 \%$ of total tuberculosis cases, and it could be as high as $50 \%$ to $70 \%$ in AIDS patients and often coexisting with pulmonary disease. Abdominal tuberculosis is the sixth most common site of extrapulmonary tuberculosis; in the range of $11 \%$ to $16 \%$, and its incidence increases proportionally to the rising incidence of tuberculosis and HIV worldwide. ${ }^{13,14}$ In HIV positive individual abdominal tuberculosis has been reported as the most common form of extra-pulmonary disease with a rate of $74 \%$ in one series. ${ }^{15}$

Over the last three decades a number of audits of abdominal tuberculosis have been published from Southern Africa. ${ }^{16,17,18,19}$ However most of these audits are from pre-HIV era, and the relevance of these findings in the current high HIV disease prevalence is uncertain. It has been reported that hospital prevalence of HIV infection among adult surgical population is up to $39 \%$ in South Africa. ${ }^{20}$ The incidence of abdominal tuberculosis in the Western world is also increasing due to the presence of HIV and migration of population from the developing countries..$^{13,21}$ 


\subsection{Abdominal tuberculosis:}

Abdominal tuberculosis may affect the gastrointestinal tract (gastrointestinal tuberculosis), peritoneum (tuberculous peritonitis), mesenteric and retroperitoneal lymph nodes (tuberculous adenitis) and solid organs e.g. liver, spleen, kidney, pancreas. ${ }^{22,23}$ Tuberculous peritonitis was the commonest form of abdominal tuberculosis before the HIV era and had contributed to $0.1 \%$ to $1.5 \%$ of total cases of tuberculosis. ${ }^{24}$ Peritoneal tuberculosis is classified conventionally into plastic (dry) and serous (wet) types. Mildly tender abdominal masses and doughy abdomen characterize the plastic type, ascites with or without signs of peritonitis characterize the serous type. Reports from early 1980 's stated that the disease involved the intestine in $37 \%$ of cases, tuberculous ascites and plastic peritonitis accounted for a further $57 \%$ of cases, while mesenteric lymphadenitis accounted for only $6 \%$ of cases. ${ }^{16}$ The recent large series by Clarke et $\mathrm{al}^{17}$ of patients with abdominal tuberculosis demonstrated that ascites is relatively uncommon and abdominal lymphadenopathy is a prominent feature and the authors also found that heterogenous complex retroperitoneal, abdominal masses were a common feature of abdominal tuberculosis.

Table 1 is taken from a recent report which summarizes and compares all the relevant audits of abdominal tuberculosis, and how it was diagnosed. ${ }^{17}$ 
Table 1: Comparison of the frequency of the various diagnostic criteria in the reported series. ${ }^{17}$

\begin{tabular}{|c|c|c|c|c|c|}
\hline Parameter & Novis $^{25}$ & Gunn $^{26}$ & Gilinsky $^{16}$ & Uygur-Bayramicli $^{27}$ & Clarke $^{17}$ \\
\hline No. of patients & 59 & 12 & 54 & 31 & 67 \\
\hline $\begin{array}{l}\text { Period of data } \\
\text { collection }\end{array}$ & $1962-1971$ & $1965-1969$ & $1972-1981$ & 1998-2001 & $2003-2005$ \\
\hline $\begin{array}{l}\text { AFB present in } \\
\text { the lesion }{ }^{a}\end{array}$ & $7(12 \%)$ & $3(25 \%)$ & $11(20 \%)$ & $5(16 \%)$ & $9(13 \%)$ \\
\hline $\begin{array}{l}\text { Caseating } \\
\text { granulomas }^{a}\end{array}$ & 14 (24\%) & $7(58 \%)$ & $9(17 \%)$ & $19(61 \%)$ & $11(16 \%)$ \\
\hline Culture $^{a}$ & $1(2 \%)$ & $2(17 \%)$ & $2(4 \%)$ & NS & Nil \\
\hline $\begin{array}{l}\text { Operative } \\
\text { description }\end{array}$ & $27(46 \%)$ & $4(33 \%)$ & $5(9 \%)$ & NS & $14(21 \%)$ \\
\hline $\begin{array}{l}\text { Evidence of TB } \\
\text { elsewhere }\end{array}$ & $10(17 \%)$ & NS & $19(35 \%)$ & NS & $2(3 \%)$ \\
\hline $\begin{array}{l}\text { Response to } \\
\text { treatment alone }\end{array}$ & Nil & Nil & $5(9 \%)$ & $9(29 \%)$ & $45(67 \%)$ \\
\hline
\end{tabular}

AFB: acid-fast bacilli; NS: not stated.

${ }^{a}$ These criteria refer to the histologic and microbiologic findings in the resected lesion.

The comparison shows that HIV/AIDS has resulted in a resurgence of abdominal tuberculosis in South Africa. In addition the current clinical presentation of abdominal tuberculosis is different from the past. The disease frequently presents as an acute or semi urgent surgical referral with underlying chronic illness. Previously patients used to present with a primary surgical pathology and 
HIV infection as a co-morbidity, but now increasingly patients present primarily with AIDS and AIDS related pathology.

\subsection{Diagnosis of abdominal tuberculosis:}

Establishing the diagnosis of abdominal tuberculosis has always been difficult because of vague and nonspecific clinical features, lack of efficient and sensitive diagnostic tools and the low yield of mycobacterium smear or culture. Delay in diagnosis and delay in initiation of therapy results in poorer outcome. It is very important to advocate a method that gives early diagnosis of this deadly disease, especially when existing treatment has been proven to be very effective and there is good evidence to suggest detrimental effects of delayed treatment. Treatment delay has been proven to be the most significant factor of attributable to mortality from abdominal tuberculosis. ${ }^{28}$ Mortality rate can be as high as $60 \%$ if anti tuberculosis treatment is not started within 30 days of symptom onset. ${ }^{29}$ There has been a trend to make use of empirical trials of anti-tuberculosis therapy to establish the diagnosis. This is problematic as the definition of clinical recovery is vague and there are different diagnoses such as lymphoma or malignancy that can mimic abdominal tuberculosis. ${ }^{8,17,30}$ In addition commencing patients on unnecessary anti tuberculosis therapy exposes them to the risk of drug interactions and side effects without any benefit. Since the early 1980's a diagnostic model has been widely used in South Africa to make the diagnosis of abdominal tuberculosis; the model included hard and soft criteria. ${ }^{16,25}$ 


\section{The hard criteria include:}

- Microbiological or histological evidence of Mycobacterium tuberculosis

- Granulomas with caseous necrosis

- Successful culture of Mycobacterium tuberculosis from the tissue specimen

- Evidence of tuberculosis at a distant site

- Typical operative findings in conjunction with macroscopic caseation and caseating granulomas with or without acid-fast bacilli (AFB) on histology

- Clinical diagnosis at autopsy

These hard criteria generally required an operative procedure with tissue resection or colonoscopic examination to provide histological or microbiological evidence of tuberculosis.

\section{The soft criteria include:}

- Clinical features

- Radiological features

- Response to chemotherapy without recurrence. 


\subsection{Traditional methods of TB diagnosis:}

If biopsy of palpable peripheral lymph node demonstrates caseating granuloma or the presence of Mycobacterium tuberculosis, the diagnosis is established and treatment can be started. This is hard evidence of tuberculosis. In the absence of easily accessible lesions we look for supportive evidence e.g. radiologic evidence or analysis of ascitic fluid. Abnormal chest X-rays, elevated erythrocyte sedimentation rate (ESR) and normocytic normochromic anaemia often accompany abdominal tuberculosis, but these tests are so non-specific that they have very little diagnostic importance.

\subsection{Alternate diagnostic adjuncts for TB:}

Serum C-reactive protein (CRP) is an acute phase reactant synthesized by the hepatocytes under the influence of interleukin-6 arising at sites of infection, inflammation and trauma. High levels of CRP are caused by infections, malignancies, chronic inflammatory diseases and trauma. But a CRP test cannot show where the inflammation is located or what is causing it. Serum concentrations of CRP increase within six hours of induction of an inflammatory process and due to its very short half live in the circulation, the initially elevated CRP levels return to normal value after resolution of the inflammatory process. Though CRP values can never be diagnostic on their own and can only be interpreted at the bedside, in full knowledge of the other clinical and pathological results, it has been used both as diagnostic and prognostic tool for tuberculosis. ${ }^{31,32,33}$ 
Ascitic fluid can be aspirated only if there is clinically evident ascites. Most of the patients currently presenting with abdominal tuberculosis do not have ascites and they present with plastic (dry) type. Hence, there is no overt fluid to aspirate and evaluate to establish the diagnosis. If aspirated fluid is shown to be exudative in nature with lymphocytes predominant; it is only suggestive of tuberculosis, not confirmatory. Direct smear for Ziehl-Neelsen stain is unhelpful most of the time, with reported sensitivity ranging from $0 \%$ to $6 \% .{ }^{24}$ The frequency of a positive culture for mycobacterium from small volumes of ascitic fluid has been less than $20 \%$ and it takes considerable time before results are available, although the positive rate can be improved by obtaining one litre of ascitic fluid concentrated by centrifugation. ${ }^{34}$

The role of ascitic fluid adenosine deaminase activity (ADA) has been studied to differentiate tuberculosis from other causes of ascites. ADA is an enzyme widely distributed in tissues and body fluids and the most important biologic activity is related to lymphoid tissues, because ADA is necessary for proliferation and differentiation of T-Lymphocytes. It has been suggested that an increasing ADA activity relates to the intensity of stimulation and the maturation state of the lymphocyte, due to the immune cellular response against Mycobacterium tuberculosis. ${ }^{13}$ Studies from a tuberculosis endemic area like South Africa have reported sensitivity and specificity exceeding $92 \%$ for this non-invasive test, ${ }^{35}$ whereas the study from the United States showed that the ascitic fluid ADA 
activity has good accuracy but poor sensitivity and imperfect specificity, where the prevalence of tuberculosis is low and underlying cirrhosis is common. ${ }^{36}$

Gamma ( $\mathrm{Y}$ ) interferon, secreted by antigen-triggered CD4+ lymphocytes, is a key lymphokine that activates macrophages, increasing their bactericidal activity against Mycobacterium tuberculosis. ${ }^{34}$ Although the sensitivity and specificity of ascitic fluid ADA and $y$-interferon activity are high in tuberculous peritonitis, their activities have been reported to be significantly lower in patients with low ascitic fluid protein concentration and in patients with AIDS, explained by a low lymphocyte activity due to the CD4+ lymphocyte depletion in HIV infection. ${ }^{13,34}$

Serum CA-125 level may be raised in tuberculous peritonitis, but the test is not specific because other conditions can give rise to the level e.g. carcinoma of the ovaries, though the decreasing CA-125 level is useful to evaluate the efficacy of therapy in TB peritonitis. ${ }^{37}$

\subsection{Newer modalities to diagnose TB:}

The yield of polymerase chain reaction (PCR) in the diagnosis of tuberculosis is high in tissues, but not in ascitic fluid; the specimens need to be fresh, the test is costly and above all the only way to get the tissue is either by laparoscopy/ laparotomy or colonoscopy. ${ }^{38}$ PCR could efficiently complement conventional bacteriological tools for the rapid diagnosis of tuberculosis but cannot replace them. ${ }^{39}$ 
A recently developed RD-1 gene-based assay for diagnosing tuberculosis infection shows promising results. Serological tests were performed to evaluate the interferon- $\mathrm{y}$ producing $\mathrm{T}$-cell response using peripheral blood mononuclear cells in patients with suspected abdominal tuberculosis and the results suggest that the tests are useful adjunct to the current tests for diagnosing abdominal tuberculosis. ${ }^{40}$ The test is called "Quantiferon Gold test" and is an enzyme-linked immunospot (ELISpot) assay which detects the release of interferon-gamma (IFN-g) in fresh heparinized whole blood from sensitized persons when it is incubated with mixtures of synthetic peptides simulating two proteins present in Mycobacterium tuberculosis. Though the sensitivity of detecting tuberculosis infection in persons with untreated culture-confirmed tuberculosis is approximately $80 \%$, sensitivity for particular groups of tuberculosis patients (e.g., young children, immunocompromised patients with HIV infection and patients with immunosuppressive drugs) has not been determined. ${ }^{41}$ The other drawback of this test is: it has only role in diagnosing latent tuberculosis in immune competent patients and cannot distinguish active from latent disease. 


\subsection{Imaging in the diagnosis of abdominal tuberculosis:}

\subsubsection{Ultrasonography (U/S):}

The traditional ultrasonic features of abdominal tuberculosis e.g. enlarged mesenteric lymph nodes of greater than $15 \mathrm{~mm}$ with hypoechoic or necrotic area, solid organ abscesses or hypoechoic lesions especially in the spleen, bowel wall thickening and ascites are well documented. ${ }^{42}$

Besides intra-abdominal fluid and lymphadenopathy, "club sandwich" or "sliced bread" and pseudokidney signs are highly suggestive of tuberculosis. ${ }^{43}$ "Club sandwich" or "sliced bread" sign is due to localized fluid between radially oriented bowel loops, due to local exudation from the inflamed bowel. Pseudokidney sign is the involvement of the ileo-caecal region which is pulled up to the subhepatic position. Though the sensitivity and specificity of $U / S$ examination have been reported to be $45 \%$ and $96 \%$ respectively in one study, ${ }^{44}$ another study showed that though the sensitivity and specificity could not be established, U/S abdomen had clinical utility in the diagnosis and treatment follow up of abdominal tuberculosis in the resource constrain areas. ${ }^{45}$

\subsubsection{Computerized tomography (CT) scans:}

CT features of abdominal tuberculosis include thickening of the small bowel mucosa due to tuberculous infiltration, stranding and thickening of small bowel mesentery, omental and retroperitoneal lymph node involvement with central caseous necrosis and peripheral rim enhancement to give a typical halo appearance. Central necrosis with rim enhancement though not pathognomonic, 
is a useful sign and readily seen in the current generation CT scanners. ${ }^{46}$ Ongoing necrotic breakdown results in large inflammatory retroperitoneal collections. CT reliably demonstrates the entire range of findings. Although peripheral rim enhancement is very characteristic of tuberculous lymphadenopathy, it is also noted in other processes such as lymphoma, metastatic malignancy, pyogenic infections and Whipple's disease. ${ }^{47}$ All the patients with abdominal tuberculosis do not present with typical CT finding. ${ }^{48}$ Although no single CT feature is diagnostic of abdominal tuberculosis, CT findings interpreted in the light of clinical and laboratory data can be a valuable tool in the diagnosis of abdominal tuberculosis. The sensitivity and specificity of CT scan in the diagnosis of TB abdomen were $92 \%$ and $95 \%$ respectively in one study, though the sample size was small ${ }^{49}$ and the sensitivity was only $69 \%$ in another study..$^{50}$

\subsubsection{Upper and lower gastrointestinal series (Barium studies):}

Tuberculosis can involve any region of the gastrointestinal tract, but in about $90 \%$ of cases it affects the ileo-caecal valve, and the adjacent ileum and colon. ${ }^{47}$ Thickening of the ileo-caecal valve and/or wide gaping between the valve and narrowed terminal ileum is "Fleischner" or "inverted umbrella sign", and localized stenosis opposite the ileo-caecal valve with rounded off smooth caecum and a dilated terminal ileum is called "purse string stenosis". In advanced disease, the caecum becomes conical and shrunken resulting in a widely open ileo-caecal valve with fixed and narrowed terminal ileum due to fibrosis, which is called 
"Stierlin's sign, and the persistent narrow stream of barium indicating stenosis is called "String sign". Although "Fleischner" or "inverted umbrella sign" and "purse string stenosis" are considered to be characteristic findings in ileo-caecal tuberculosis, "Stierlin's sign" and "String sign" are not specific of intestinal tuberculosis and may also be found in Crohn's disease. ${ }^{43,47,51}$

\subsubsection{Endoscopy:}

Upper endoscopy and colonoscopy with terminal ileoscopy are investigations of choice for the diagnosis of intestinal tuberculosis as it allows for direct visualization and tissue sampling for histology and culture, however it is limited by the accessibility of the small bowel. ${ }^{52,53}$ Diffuse involvement of the entire colon is rare and endoscopically lesions look very similar to ulcerative colitis and lesions mimicking carcinoma have also been described. ${ }^{43}$

\subsection{Laparotomy to diagnose abdominal tuberculosis:}

There is a drive to reduce the number of patients being treated for tuberculosis empirically due to the incomplete treatment or incorrect use of the drugs. This is especially true in the initial phase of anti-tuberculosis treatment as a cause of acquired multi-drug resistance (MDR) tuberculosis and severe hepatotoxicity occasionally causing acute liver failure and death. ${ }^{54}$ The mortality rate among patients with anti-tuberculosis treatment associated acute liver failure was high $(67 \%)$ and $63 \%$ of patients in that study were prescribed anti-tuberculosis

treatment empirically. ${ }^{55}$ Trials of TB treatment have been abandoned and the 
current recommendation is that when the decision is taken for empiric TB treatment, then a full course should be given. A trial of TB treatment that is prematurely stopped in a patient who does have TB may contribute to drug resistance. We find it very difficult to establish a definitive diagnosis in patients with abdominal tuberculosis. This is because patients suffering from HIV disease and tuberculosis, a formal laparotomy which is required to obtain tissue for histological or microbiological analysis is associated with significant morbidity and potential high mortality. ${ }^{17,56}$ Furthermore the volume of patients with this problem would mean that resorting to laparotomy would place a great strain of resources. In the face of this high volume of chronically sick patients the general surgeons have resorted to commencing empirical trials of therapy and waiting for a response to therapy. There are problems with this approach. There is no standardization of what is meant by a positive response to treatment or what constitutes an adequate trial of therapy is also unclear; the weight gain, resolution of constitutional symptoms, improvement of haemoglobin percentage and reduction in CRP levels may be considered as indicators of response to therapy. Trial of therapy may cause delay in the diagnosis of other diseases which mimic abdominal tuberculosis e.g. Crohn's disease, lymphoma, malignancy and other opportunistic infections. ${ }^{51,57,58,59,60}$

Until recently laparotomy was the only available diagnostic procedure to obtain adequate tissue to make a definitive diagnosis of abdominal tuberculosis. Many patients who present with signs and symptoms of an acute abdomen are 
subjected to emergency laparotomy. Laparotomy in these patients may result in significant associated morbidity. It is important to avoid emergency surgery in patients without radiologic or clinical evidence of free perforation or disseminated peritonitis and in immunocompromised patients with significant co-morbidities. Although elective laparotomy does not carry an unduly high mortality rate, in emergency laparotomy mortality rate can be as high as $60 \% .{ }^{17}$ The most significant complications of laparotomy in patients with abdominal tuberculosis are anastomotic leak and fistula. It has been reported that enterolysis is the main cause of fistula formation and it is concluded that when a surgeon encounters tuberculosis at laparotomy, it would be advisable to avoid any enterolysis and to confine the procedure in taking specimens for microbiological and histopathological examinations, then the incision would be closed and the patient put on appropriate anti-TB medications. ${ }^{61}$

\subsection{History of laparoscopy in diagnosis of abdominal tuberculosis:}

Diagnostic peritoneoscopy with a direct optic scope was used in the 1960's in patients with tuberculous peritonitis to obtain peritoneal biopsy for microbiological or histological examination. Peritoneal biopsy gives a better diagnostic value than ascitic fluid analysis alone. ${ }^{18}$ 
Blind percutaneous peritoneal biopsy used to be under taken with local anaesthesia with varying degrees of success. Peritoneal biopsy was first performed by Donohue in 1959 using Vim Silverman needle and later Abrams and Cope needles were used. ${ }^{62,63}$ There are several other small series of peritoneal biopsy in the 60's, 70's and 80's. ${ }^{57,64,65}$ The procedure carries a high risk of complications like bowel perforation, bleeding, and even death. ${ }^{66,67,68}$ The main contraindication to blind percutaneous peritoneal biopsy is the absence of ascites. The role of blind percutaneous biopsy is limited in the cases of plastic (dry) type of abdominal tuberculosis which currently predominate. ${ }^{18,62,69}$

The difficulty in confirming the diagnosis, the considerable morbidity and mortality associated with formal laparotomy in patients with abdominal tuberculosis and HIV co-infection, and the reluctance to embark on poorly defined empirical therapy have generated interest in the use of laparoscopy to obtain specimens for histological and microbiological assessment. ${ }^{17,56,61}$ The advent of laparoscopy has allowed surgeons to visually inspect the abdominal cavity and take biopsies with minimal morbidity. Laparoscopy as an aid to diagnosis is well established in the management of both chronic and acute abdominal pain. There have been interests expressed in the use of laparoscopy to establish the diagnosis of abdominal tuberculosis..$^{21,69,70,71,72,73}$ Table 2 shows the different laparoscopic series with their diagnostic yields. 
Table 2: Different laparoscopic series with their yields.

\begin{tabular}{|c|c|c|c|c|c|}
\hline Authors & Series date & $\begin{array}{l}\text { No. of } \\
\text { patients }\end{array}$ & $\begin{array}{c}\text { Ascites } \\
(\%)\end{array}$ & HIV+ & $\begin{array}{c}\text { Diagnostic yield } \\
(\%)\end{array}$ \\
\hline Mohamed $^{70}$ & 2004-2008 & 13 & 100 & not stated & 85 \\
\hline Meshikhes $^{73}$ & 1992-2008 & 20 & 100 & not stated & 75 \\
\hline Krishnan $^{71}$ & 1999-2005 & 41 & 70 & not stated & 80 \\
\hline Al-Mulhim ${ }^{21}$ & 1995-2002 & 21 & 67 & none & 81 \\
\hline $\mathrm{Rai}^{72}$ & 1995-2001 & 25 & 32 & not stated & 92 \\
\hline Mimica $^{69}$ & $1977-1987$ & 32 & 75 & not stated & 85 \\
\hline
\end{tabular}

The risk involved with laparoscopy is low but there is a definite incidence of complications which may be significant. Most of the complications associated with laparoscopy involve iatrogenic injury to major vessels or to hollow viscera. With modern techniques to safely induce a pneumoperitoneum and to safely introduce operative ports the risk is less than $0.05 \%{ }^{66}$ Patients undergoing routine laparoscopic surgery face the same risk. The patient will also have to undergo a general anaesthetic. Once again the risks associated with modern general anaesthesia are extremely low. From retrospective studies it has been postulated that in patients suspected to have abdominal tuberculosis without evidence of extra-abdominal disease, early laparoscopy may be useful to establish a histological diagnosis with acceptably low morbidity. ${ }^{71}$ Although there are some publications of retrospective studies about the role of laparoscopy to establish the diagnosis of abdominal tuberculosis, there is no prospective 
study. ${ }^{74,75,76}$ However, locally it was found that the use of laparoscopy in the diagnostic work up of suspected abdominal tuberculosis was poor and underutilized. ${ }^{17}$ Establishing a dedicated team that will aggressively pursue a diagnosis of abdominal tuberculosis with laparoscopy is an attractive concept at a busy hospital in South Africa with high incidence tuberculosis and HIV infection. This will hopefully improve patient care and allow us to establish the diagnostic accuracy of laparoscopy for this condition. 


\section{Hypothesis to be tested}

Laparoscopy is a minimally invasive procedure which will enable a definitive diagnosis of abdominal tuberculosis to be established in patients with clinically suspected but microbiologically or histologically unconfirmed abdominal tuberculosis. These patients would be eligible for empiric anti-tuberculosis treatment in terms of current standard of care. 


\section{Aims of the study}

This study aimed to review several objectives in light of the HIV-tuberculosis coinfection pandemic in diagnosing abdominal tuberculosis:

1) To quantify and establish the role of diagnostic laparoscopy in the work up of patients with suspected abdominal tuberculosis.

2) To establish the gross laparoscopic features of abdominal tuberculosis.

3) To assess the capability of laparoscopy to provide adequate microbiological and histological specimens for analysis.

4) To audit the clinical response of patients with abdominal tuberculosis to appropriate therapy. 


\section{Methodology}

A prospective clinical audit of the use of diagnostic laparoscopy in patients with suspected abdominal tuberculosis was conducted. The study has been approved by the Biomedical Research Ethics Committee (BREC) of the University of KwaZulu Natal. The study was undertaken at Edendale Hospital in Pietermaritzburg. All patients with clinically suspected but histologically or microbiologically unconfirmed abdominal tuberculosis were referred to the investigating team. The investigating team consisted of a general surgeon and an infectious disease physician. The patients then were jointly assessed by the investigating team. If a definitive diagnosis of extra abdominal tuberculosis could be achieved by another procedure e.g. sputum analysis, peripheral lymph node biopsy or fluid or lymph node aspiration; the patients were commenced on anti-tuberculosis treatment and excluded from this study cohort.

In order to maximize the diagnosis of extra abdominal tuberculosis, all study participants also had:

- Induced sputum or tissue aspirate (if any) for tuberculosis culture

- Microbiological blood culture

- Pre-operative serum CRP level

The two clinicians reviewed abdominal U/S or CT scans reports if they were done. If the clinicians agreed that clinical and radiological features were suggestive of abdominal tuberculosis and the histological or microbiological 
evidence of tuberculosis could not be obtained from any other site then the patients were offered a diagnostic laparoscopy.

Informed consents were taken from the patients. Moribund patients who are not expected to survive an anaesthetic were excluded from the study. All anaesthetic administered at Edendale Hospital are administered by trainees under direct consultant supervision. Pre-operative body weight, haemoglobin $(\mathrm{Hb}) \%$ and CRP level were recorded in all patients especially to compare with post-operative response to treatment.

All the patients undergoing laparoscopy were started on standard antituberculosis treatment without delay, whilst awaiting histology report. Positive diagnosis of abdominal tuberculosis was considered if there was: typical tuberculous granulomata containing Langhan's giant cells with caseation or non caseating necrosis with the demonstration of AFB in the biopsied tissues or the isolation of Mycobacterium tuberculosis by culture from the ascitic fluid or biopsied tissues. This equates to "Hard criteria" and as such if one can reliably establish them then abdominal tuberculosis can be treated correctly. The surgeon prospectively followed up each patient enrolled in the study until the point at which we have confirmed a diagnosis of tuberculosis and evaluated the response of the treatment, which was at the end of eight weeks. If histological or microbiological confirmation of abdominal tuberculosis were obtained from laparoscopy then the patients were referred for follow- up to the tuberculosis 
clinic at Edendale Hospital. The data were evaluated to see the diagnostic yield of laparoscopy in the form of macroscopic appearance and histological or microbiological results and the response to medications in the form of sensitivity and specificity.

All participants were to evaluated for tuberculosis treatment response by assessment at week 4 and week 8 following laparoscopy. Therapeutic response was considered if there were at least two or more of the following criteria present: weight gain $\geq 5 \%$, haemoglobin increase $\geq 1 \mathrm{gm} \%, 60 \%$ reduction in CRP, and at least half of the symptoms were much better or resolved, including assessment of adherence to tuberculosis treatment using the TB clinic "Green Card" were evaluated. All the patients had repeat abdominal U/S at follow up to compare the findings with the preoperative U/S reports.

\section{Inclusions criteria:}

In essence there were two broad groups of patients who were considered for laparoscopy:

1) Those with abdominal pain with mild peritonism and features compatible with HIV disease (or known to be HIV sero-positive) and a history of weight loss with drenching sweats for more than two weeks (dry type).

2) Those with ascites and features compatible with HIV disease (or known HIV sero-positive) and history of weight loss with drenching sweats for more than two weeks (wet type). 


\section{Exclusions criteria:}

The patients were excluded from the study in the case of:

1) Moribund patients not fit for anaesthetic.

2) Patients with histologically or microbiologically confirmed TB abdomen from laparotomy for other reasons or ascitic fluid examinations

3) Informed consent not obtained

4) Minors less than 16 years of age 


\section{Laparoscopic technique}

Laparoscopy was done under general anaesthesia in all patients. The first 10 $\mathrm{mm}$ trocar was introduced in the subumblical region under direct vision using visiport in most of the patients to avoid bowel injuries due to adhesions. Verese needle was used in only a few cases to insufflate the peritoneal cavity before inserting the trocar. A second $5 \mathrm{~mm}$ trocar was introduced under direct vision in the suprapubic region. A third $5 \mathrm{~mm}$ or $10 \mathrm{~mm}$ trocar was introduced under direct vision according to the abnormalities found; most of the cases in the left iliac fossa. In most of the cases the abnormalities were found around the ileo-caecal region and were possible to biopsy with these three ports. Whole abdominal cavity was inspected: if there was any free fluid, it was aspirated and sent for microbiological examination, any abnormal looking tissues e.g. enlarged lymph nodes, mass, omentum, peritoneum or liver tissues were biopsied and sent for histological examinations. Due to a persistent logistic problem with the processing and delivery of tissue specimens for tuberculosis culture, only three specimens were deemed suitable for culture and hence no analysis of this technique was carried out. If the macroscopic feature was not suggestive of tuberculosis tru-cut biopsy of liver was done. Trocar sites were closed with nonabsorbable sutures. 


\section{Assessing diagnostic accuracy}

In assessing the diagnostic accuracy of a test or a group of tests using sensitivity and specificity as in this study, the gold standard definition of abdominal tuberculosis is key to defining the true positives. We appreciate that there are significant advances in diagnostic tests in pulmonary tuberculosis and in the diagnosis of disseminated tuberculosis, which have been discussed in the introduction. However the possibility of dual diagnosis of tuberculosis and another pathology led us not to focus purely on the diagnosis of tuberculosis but on the establishment of the diagnosis of abdominal tuberculosis. This was because we also wanted to establish if there was an alternate or metachronous diagnosis which would require specific therapy. Therefore our study focused on abdominal tissue and fluid sampling. We therefore chose the three-criteria stated in the statistical section as the gold standard. 


\section{Statistical analysis}

Statistical evaluation of data entailed sensitivity (Se), specificity (Sp), positive predictive value (PPV) and negative predictive value (NPV) analysis. The "gold standard" for the diagnosis of abdominal tuberculosis was (1) presence of AFB in histological specimens, (2) presence of caseating granulomas in histological specimens and (3) TB culture.

In the case of quantitative data, means and $95 \%$ confidence interval $(95 \% \mathrm{Cl})$ were reported around sample estimates. MS Excel and Epicalc 2000 (Joe Gilman and Mark Myatt 1998, Brixton Books) were used to analyze the data. 


\section{Results}

From January 2008 to June 2010 a total of 190 patients were referred to us with a provisional diagnosis of abdominal tuberculosis and no hard evidence of tuberculosis at a site other than the abdomen e.g. negative sputum for AFB, no positive culture from any aspirate. The mean age of the whole group was 33 years (95\% Cl $31-34$ years). 94 patients were male and 96 were female. The patients were divided into three groups: Group A- laparoscopy, Group Blaparotomy, Group C- no surgical intervention. Figure 1 shows the distribution of all the patients in the study.

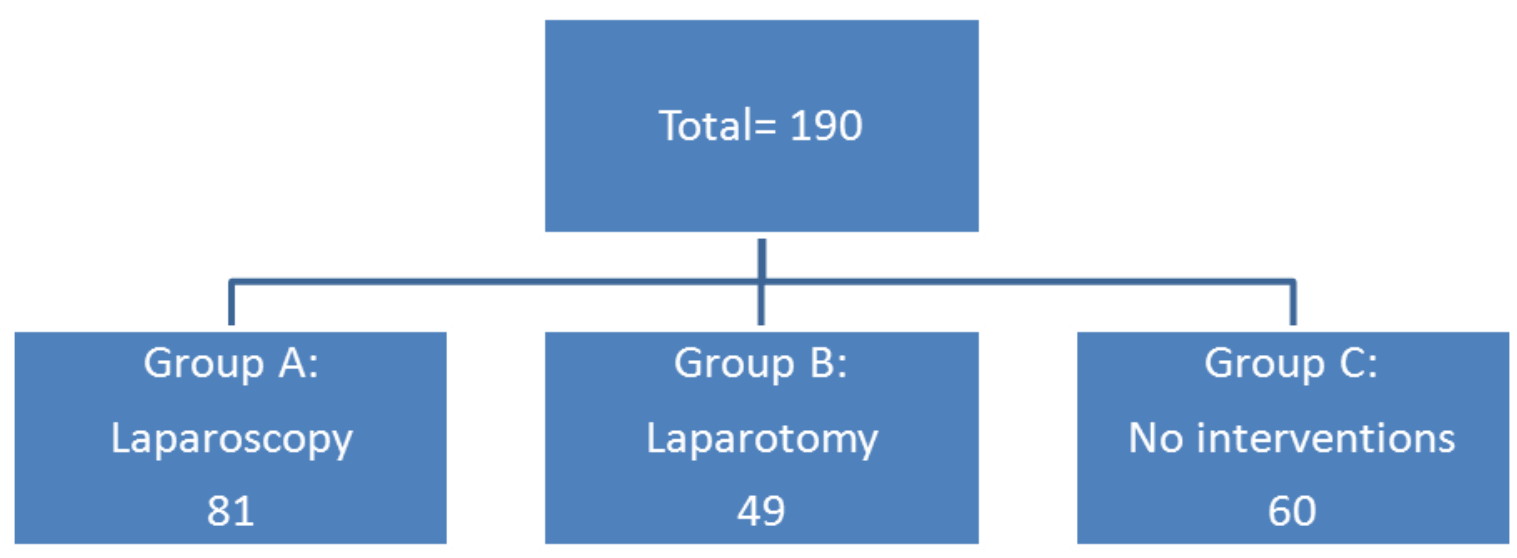

Figure 1: Distribution of all the patients in the study. 


\subsection{Group A: Laparoscopy}

Eighty one patients had diagnostic laparoscopy, 34 of them were male and 47

were female (male: female=1:1.38), mean age was 33 years $(95 \% \mathrm{Cl} 31-36$ years). Only two patients were Indian origin and the rest were African. Sixty two patients were HIV positive (77\%); only 10 of them had known CD4 counts and the mean counts were $138(95 \% \mathrm{Cl} 57-210)$ and 16 patients were on antiretroviral therapies, six patients were HIV negative (7\%) and 13 had an unknown HIV status (16\%) because they declined HIV testing.

\subsubsection{Duration of symptoms and hospital stays:}

The mean duration of symptoms was 41 days ( $95 \% \mathrm{Cl} 31$ - 51 days) prior to the hospital admission and the mean duration of hospital stay was 11 days $(95 \% \mathrm{Cl}$ 10- 13 days).

\subsubsection{Clinical presentation:}

The most common clinical features were abdominal pain, abdominal distension, lymphadenopathy, night sweats and weight loss. Preoperative body weight were recorded in the patients. The mean body weight was $55 \mathrm{~kg}(95 \% \mathrm{Cl} 53-58 \mathrm{~kg})$. Table 3 is showing the clinical presentation of 81 patients. 
Table 3: Clinical presentation of 81 patients.

\begin{tabular}{|l|c|c|}
\hline Clinical features & Numbers & Percentage (\%) \\
\hline Abdominal pain & 81 & 100 \\
\hline Weight loss & 69 & 85 \\
\hline Night sweats & 61 & 75 \\
\hline Lymphadenopathy & 52 & 64 \\
\hline Abdominal distension & 22 & 27 \\
\hline Low grade fever & 19 & 24 \\
\hline Vomiting & 18 & 22 \\
\hline Previous lungs TB & 18 & 22 \\
\hline Constipation & 8 & 10 \\
\hline Diarrhoea & 4 & 5 \\
\hline Ascites & 2 & 2 \\
\hline
\end{tabular}

${ }^{*}$ Percent rounded to the nearest integer 


\subsubsection{Preoperative investigations:}

\subsubsection{Haemoglobin $(\mathrm{Hb}) \%$ :}

$\mathrm{Hb}$ levels were checked in all the patients. The mean $\mathrm{Hb}$ was $9.69 \mathrm{gm} \%(95 \% \mathrm{Cl}$ 9.22- $10.14 \mathrm{gm} \%)$. Nineteen patients (23\%) had $\mathrm{Hb}$ level less than $8 \mathrm{gm} \%$.

\subsubsection{White Cells Counts (WCC):}

WCCs were checked in all the patients. The mean WCC was $8.62 \times 10^{9} / \mathrm{L}(95 \%$ Cl 7.56- $\left.9.68 \times 10^{9} / \mathrm{L}\right)$. Most of the patients $(63 \%)$ had normal WCC (normal count is $\left.4-11 \times 10^{9} / \mathrm{L}\right)$.

\subsubsection{Neutrophils:}

Neutrophils counts were checked in all the patients. The mean neutrophil count was $72.22 \%$ (95\% Cl 69.17- 75.26\%). Only one patient (1\%) had neutropenia, 33 patients $(41 \%)$ had neutrophilia and 47 patients (58\%) had normal neutrophil count (normal: 40-75\%).

\subsubsection{Lymphocytes:}

Lymphocytes counts were checked in all the patients. The mean lymphocytes count was $17.6 \%(95 \% \mathrm{Cl} 15.36-19.83 \%)$. There was no patient with lymphocytosis, 41 patients (51\%) had lymphopenia and 40 patients (49\%) had normal lymphocytes count (normal: 20-45\%). 


\subsubsection{Chest X-Ray (CXR):}

CXR were done in all patients but it was suggestive of tuberculosis in only ten patients $(12 \%)$ though sputum cultures were negative in all of 10 patients. Most of the patients had non-productive cough with nonspecific pulmonary infiltrates. Sputum cultures were performed only in patients with CXR suggestive of tuberculosis or in patients with associated cough and all cultures were negative for tuberculosis.

\subsubsection{U/S abdomen:}

$\mathrm{U} / \mathrm{S}$ abdomen were done in 81 patients and all patients had evidence suggestive of abdominal tuberculosis, but histology/culture results were positive for tuberculosis in 55 patients (68\%) and no specimen was taken from one patient. Sonographic features were retroperitoneal lymphadenopathy (75), free fluid (11), complex ascites (17), thickened small bowel loops (14), retroperitoneal abscesses (3), and hypo-echoic/ necrotic echo pattern of the liver and spleen (3).

\subsubsection{CT scan abdomen:}

CT abdomen was done in 40 patients; 36 of them were suggestive of abdominal tuberculosis and four were not. Features suggestive of TB were mesenteric lymphadenopathy (38), thickened small bowel wall (12), hypodensity of liver and spleen (3), free fluid (17), and mesenteric stranding (10). Only 19 of them had positive (48\%) histology for tuberculosis and 21 were negative (52\%). The sensitivity and specificity of CT scan are $95 \%(95 \% \mathrm{Cl} 72-100 \%)$ and $14 \%(95 \%$ 
Cl 4- 37\%) respectively. The positive predictive value and negative predictive value of CT scan was $50 \%(95 \% \mathrm{Cl} 33-67 \%)$ and $75 \%(95 \% \mathrm{Cl} 22-99 \%)$ respectively. Figures 2-5 are showing CT scan findings in different patients.

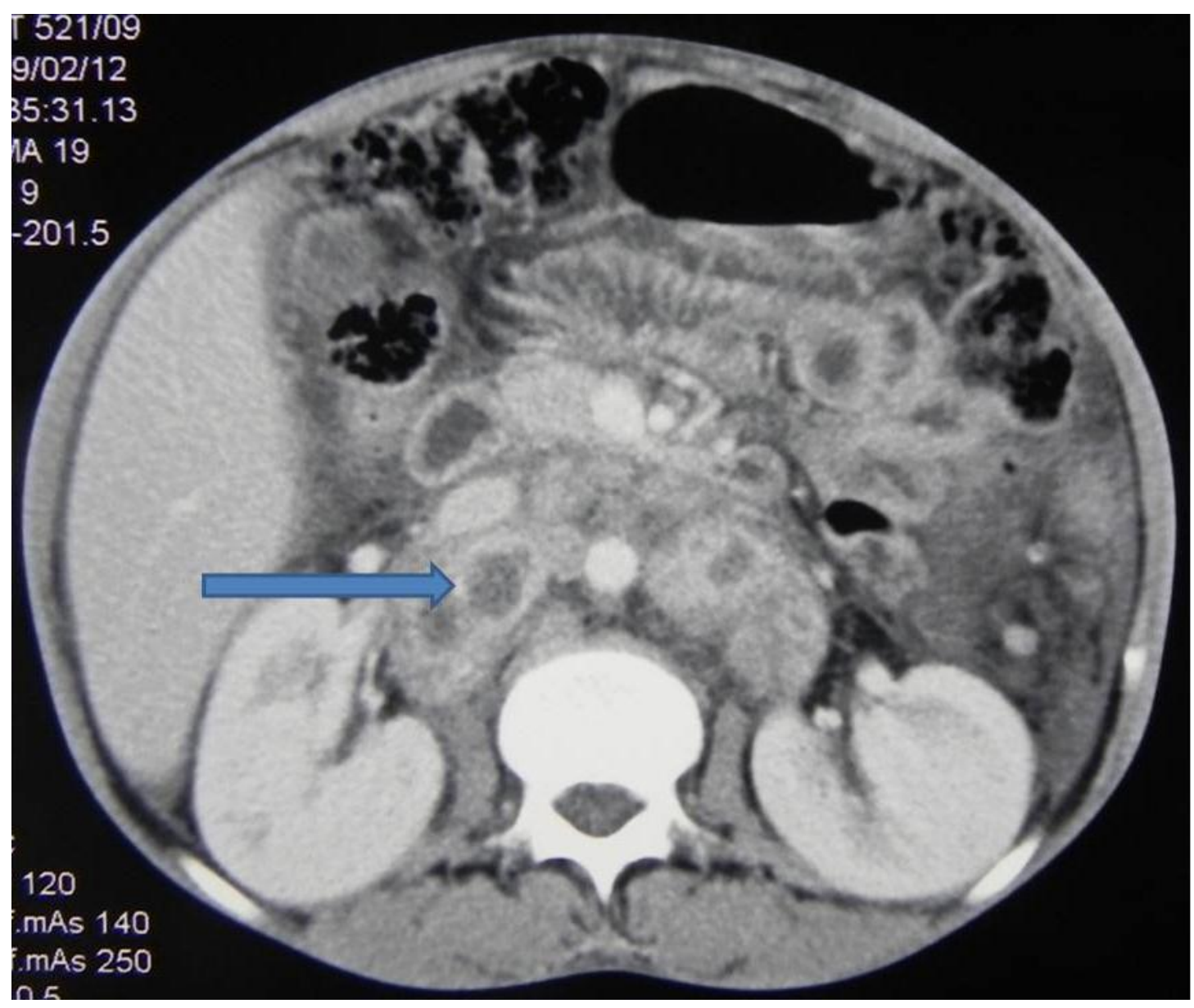

Figure 2: CT scan showing enlarged retroperitoneal lymph nodes (arrow) with central area of necrosis; histology was positive for TB. 


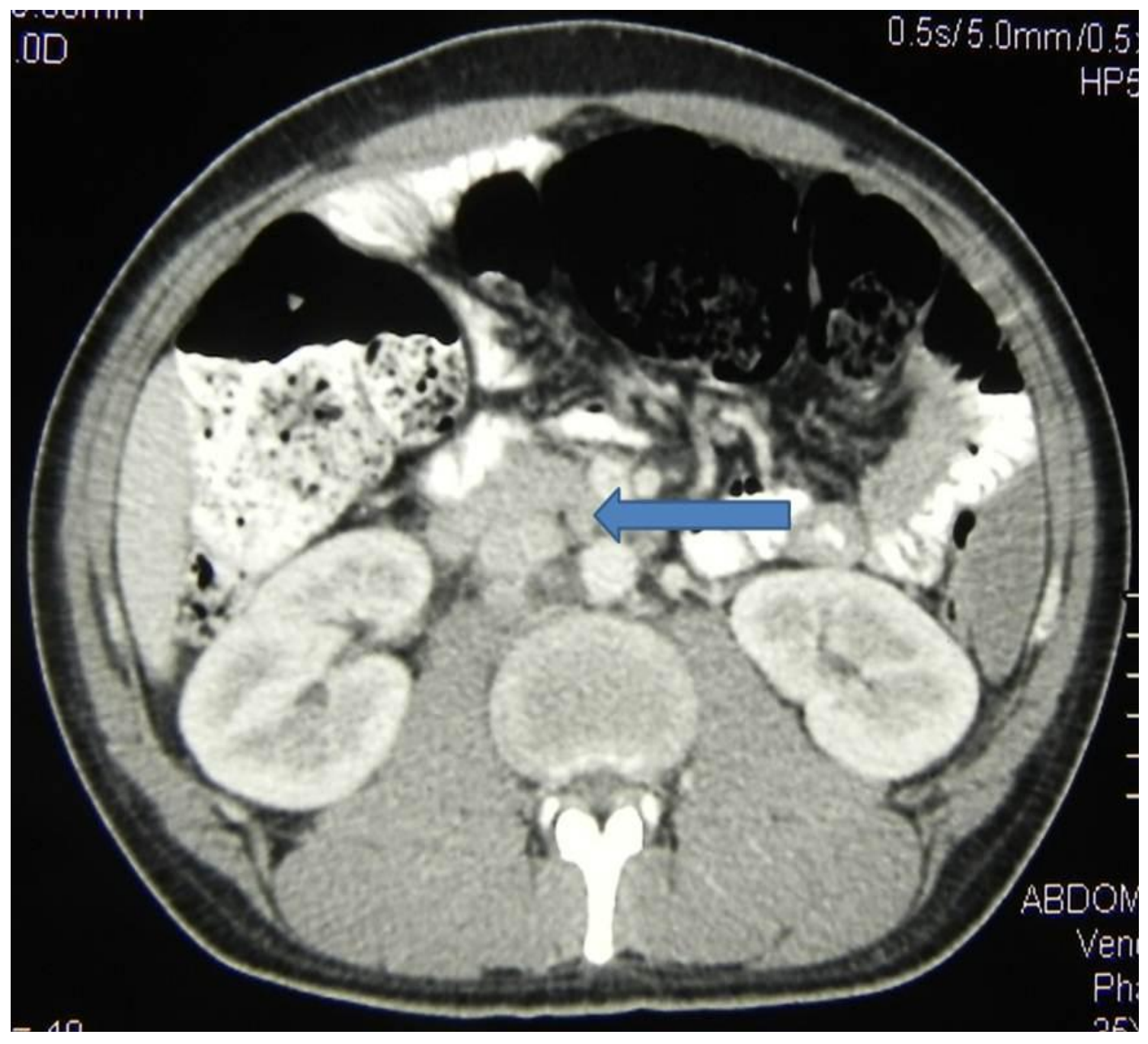

Figure 3: CT scan showing enlarged retroperitoneal lymph nodes (arrow), histology was negative for TB. 


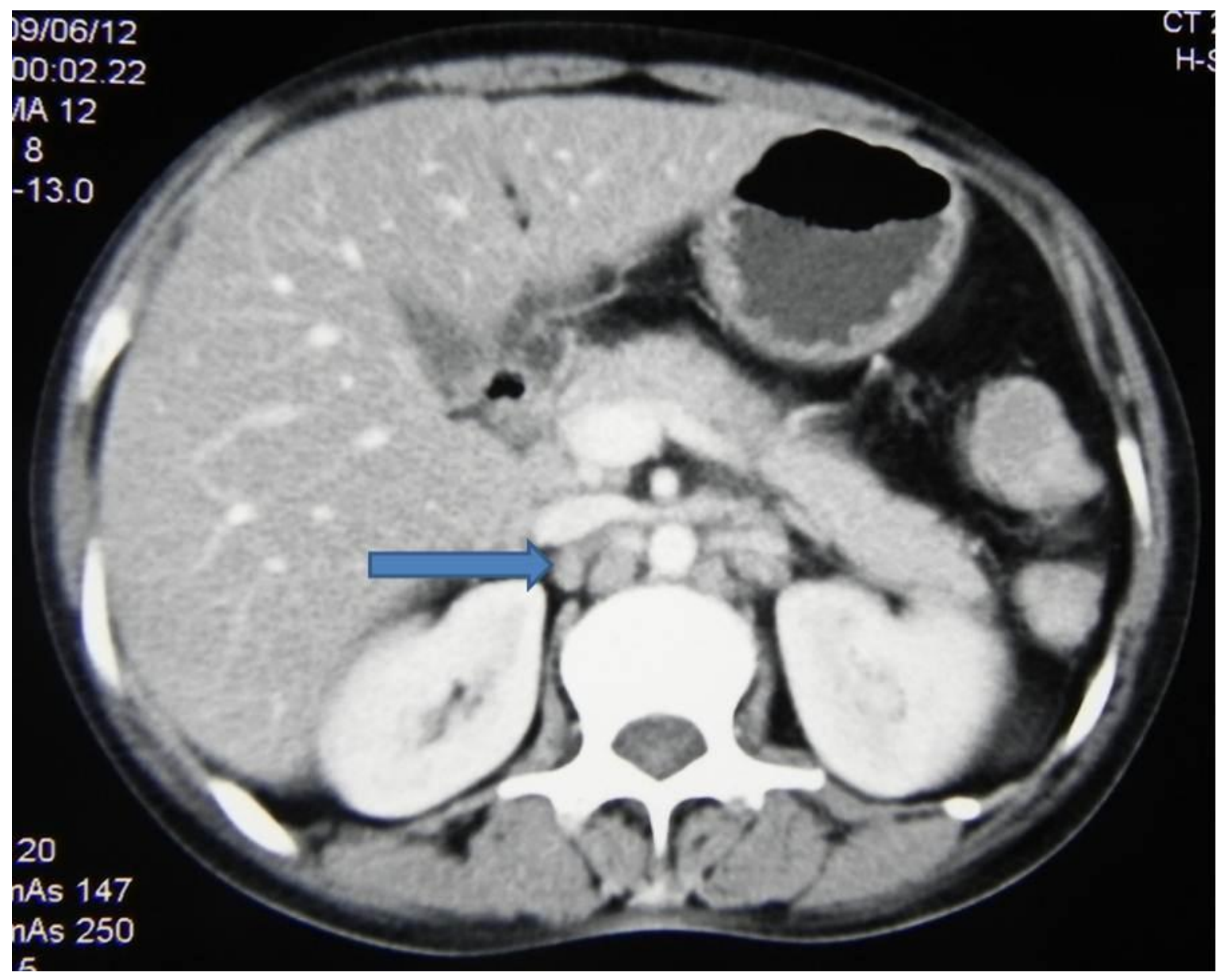

Figure 4: CT scan showing enlarged retroperitoneal lymph nodes (arrow), but no gland could be biopsied. 


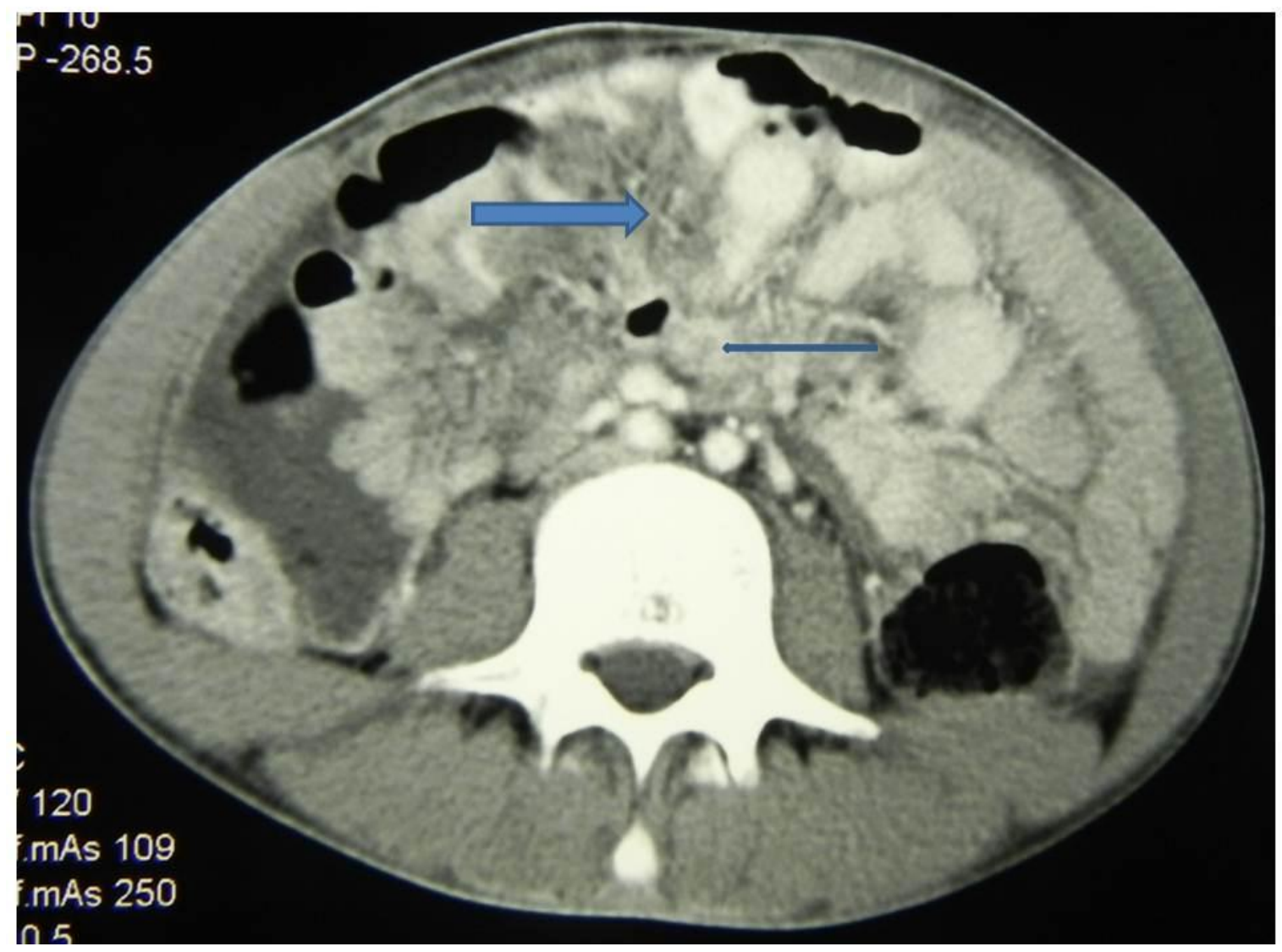

Figure 5: CT scan showing enlarged mesenteric lymph nodes (thin arrow) and thickened mesentery (bold arrow), histology showed metastatic adenocarcinoma.

\subsubsection{ESR:}

Thirty five patients had ESR done and the mean ESR was $89(13-153) \mathrm{mm}$ in $1^{\text {st }}$ hour. Only three patients (9\%) had normal ESR (less than 20) with $95 \% \mathrm{Cl} 2.24$ 24.19 and 32 had high ESR (91\%) with $95 \% \mathrm{Cl} 75.81-97.76$. Twenty two patients had positive histology/culture for tuberculosis (69\%) of the 32 patients with high ESR. The sensitivity and specificity of ESR are 95\% (95\% Cl 75- 100\%) and $17 \%(95 \% \mathrm{Cl} 3-49 \%)$ respectively. The positive predictive value and negative predictive value of ESR are $68 \%(95 \% \mathrm{Cl} 49-83 \%)$ and $67 \%(95 \% \mathrm{Cl}$ 13- 98\%) respectively. 


\subsubsection{CRP:}

CRP was done in 24 patients and the mean CRP was 68 (5-128) mg/L. Fourteen patients had positive (58\%) histology/ culture for tuberculosis, nine patients had negative histology (38\%) and no specimen was taken from one patient (4\%). CRP was normal (less than $10 \mathrm{mg} / \mathrm{L}$ ) in four patients (17\%) with $95 \% \mathrm{Cl} 5.48$ 38.19 and high in 20 patients (83\%) with $95 \% \mathrm{Cl} 61.81$ - 94.52. Eleven patients had positive histology/ culture for tuberculosis (55\%) of the 20 patients with high CRP. The sensitivity and specificity of CRP are $79 \%(95 \% \mathrm{Cl} 49-94 \%)$ and $11 \%$ (95\% Cl 1-49\%) respectively. The positive predictive value and negative predictive value of CRP are $58 \%(95 \% \mathrm{Cl} 34-79 \%)$ and $25 \%(95 \% \mathrm{Cl} 1-78 \%)$ respectively.

\subsubsection{Blood culture:}

Blood cultures for tuberculosis were done in 51 patients. Thirteen $(25 \%)$ of them had positive culture for tuberculosis and $38(75 \%)$ were negative. The sensitivity and specificity of blood culture are $38 \%(95 \% \mathrm{Cl} 21-58 \%)$ and $91 \%(95 \% \mathrm{Cl} 69$ $98 \%$ ) respectively. The positive predictive value and negative predictive value of blood culture are $85 \%(95 \% \mathrm{Cl} 54-97 \%)$ and $53 \%(95 \% \mathrm{Cl} 36-69 \%)$ respectively. Unfortunately it took 6-8 weeks to get the culture results back and it was not helpful in the diagnosis and management of the condition. 
Table 4 is showing the diagnostic yields of preoperative investigations.

Table 4: Diagnostic yields of pre-operative investigations.

\begin{tabular}{|l|c|c|c|c|c|c|c|}
\hline Investigations & $\begin{array}{c}\text { No. patients } \\
\text { (\%) }\end{array}$ & $\begin{array}{c}\text { Number } \\
\text { positive }\end{array}$ & $\begin{array}{c}\text { (\%) } \\
\text { positive }\end{array}$ & $\begin{array}{c}\text { Sensitivity } \\
(\%)\end{array}$ & $\begin{array}{c}\text { Specificity } \\
\text { (\%) }\end{array}$ & $\begin{array}{c}\text { PPV } \\
\text { (\%) }\end{array}$ & $\begin{array}{c}\text { NPV } \\
\text { (\%) }\end{array}$ \\
\hline ESR & $35(43)$ & 22 & 69 & 95 & 17 & 68 & 67 \\
\hline CRP & $24(30)$ & 14 & 58 & 79 & 11 & 58 & 25 \\
\hline CT scan & $40(49)$ & 19 & 48 & 95 & 14 & 50 & 75 \\
\hline Blood culture & $51(63)$ & 13 & 25 & 38 & 91 & 85 & 53 \\
\hline
\end{tabular}

$\mathrm{PPV}=$ positive predictive value, $\mathrm{NPV}=$ negative predictive value

*Percent rounded to the nearest integer

The sensitivity, specificity, PPV and NPV calculations were based on the number who had the sample taken, and not on the total number (81) undergoing laparoscopy.

\subsubsection{Endoscopies:}

Upper gastrointestinal (GI) endoscopies were done in 10 (12\%) patients and colonoscopies were done in nine $(11 \%)$ patients, but none yielded any specific diagnosis.

\subsubsection{Contrast studies:}

Two patients (2\%) had barium enema and three patients (4\%) had upper Gl series done, which were not significant. 


\section{Laparoscopic views}

The typical appearance of abdominal tuberculosis is of ascites with multiple small deposits (tubercles) of about $0.5 \mathrm{~cm}$ in diameter adhering to the peritoneum and bowel walls and omentum, or there may be thin, filmy multiple white adhesions attaching the peritoneum to the bowel, omentum and liver or a deep abdominal mass from the involved mesenteric lymph nodes or thick oedematous omentum (omental cake).

\subsection{Lymph nodes:}

Mesenteric lymph nodes were biopsied from 56 patients (70\%) and 43 had positive $(77 \%)$ histology for tuberculosis. Thirteen lymph nodes $(23 \%)$ were negative for tuberculosis and 24 patients (30\%) did not have any lymph node, no specimen was taken from one patient. Amongst the 43 histology positive patients $35(81 \%)$ had caseating granuloma and eight had non-caseating granuloma (19\%), and 24 amongst all of them (56\%) had acid fast bacilli (AFB) positive. Amongst the 13 histology negative patients, 10 had nonspecific chronic inflammation with reactive lymph nodes (three had sinus histiocytosis), two had other diagnosis (adenocarcinoma and lymphoma) and one did not show any pathology.

The sensitivity and specificity of lymph nodes are $83 \%(95 \% \mathrm{Cl} 69-91 \%)$ and $54 \%(95 \% \mathrm{Cl} 34-72 \%)$ respectively. The positive predictive value and negative 
predictive value of lymph nodes are $77 \%(95 \% \mathrm{Cl} 63-87 \%)$ and $63 \%(95 \% \mathrm{Cl}$ 41- $80 \%$ ) respectively. Figure 6 is showing mesenteric lymph node with pus.

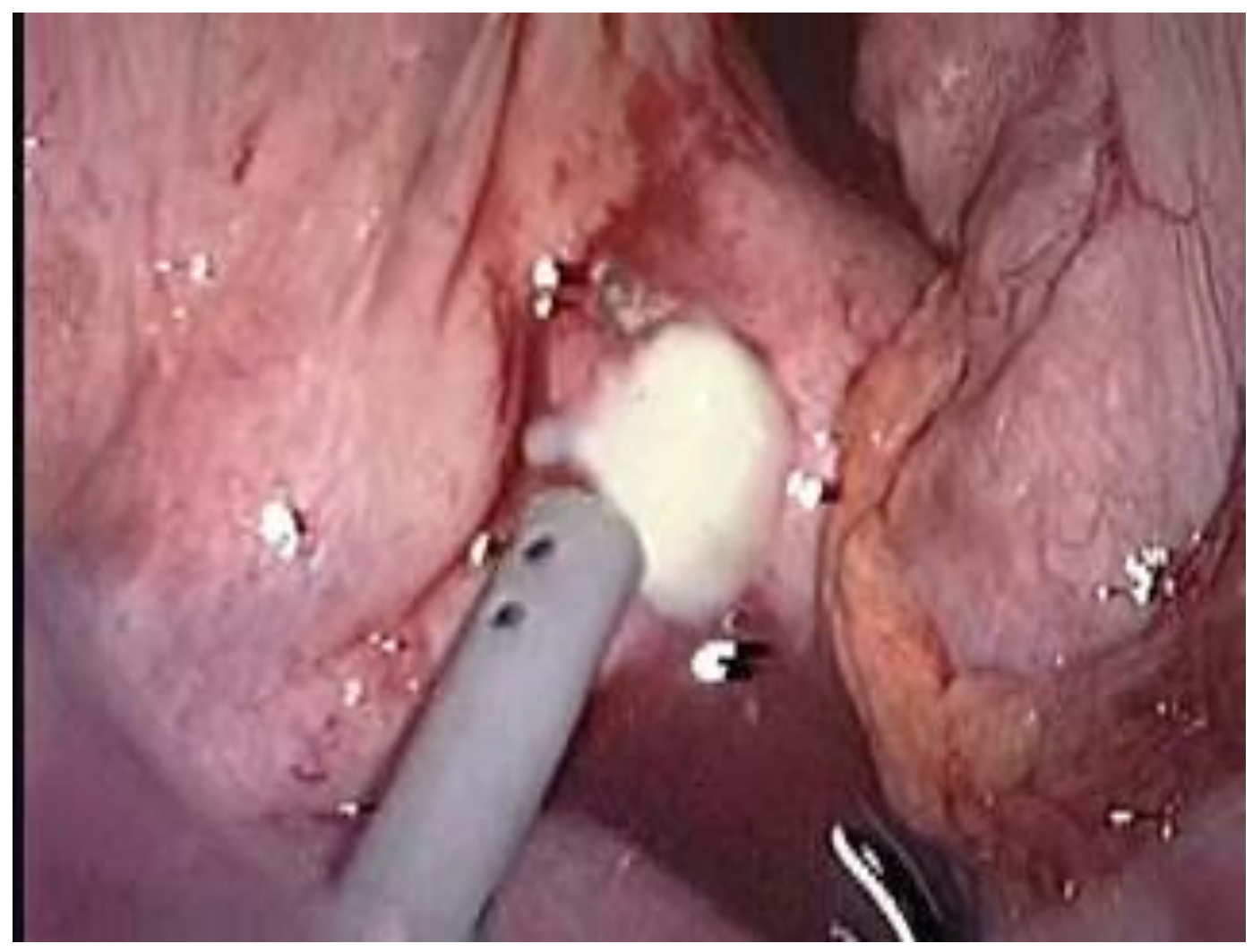

Figure 6: Mesenteric lymph node with pus 


\subsection{Ascitic fluid culture:}

Small amounts of ascitic fluid was aspirated and sent for culture from 46 patients (58\%) and ascitic fluid was absent in other 34 patients (42\%). Sixteen of them were positive (35\%) for tuberculosis culture and $30(65 \%)$ were negative. The correlation has been established between the ascitic fluid culture and histology results. It has been found that 13 patients had both fluid culture and histology positive, three patients had fluid culture positive but histology negative, 15 patients had fluid culture negative but histology positive, and 15 patients had both fluid culture and histology negative. The sensitivity and specificity of ascitic fluid culture are $46 \%(95 \% \mathrm{Cl} 28-66 \%)$ and $83 \%(95 \% \mathrm{Cl} 58-96 \%)$ respectively. The positive predictive value and negative predictive value of ascitic fluid culture are $81 \%(95 \% \mathrm{Cl} 54-95 \%)$ and $50 \%(95 \% \mathrm{Cl} 32-68 \%)$ respectively.

Only two patients had drug resistant tuberculosis: one was resistant to Isoniazid (INH) and the other was resistant all drugs except INH and Rifampicin. Figure 7 shows straw coloured pelvic fluid. 


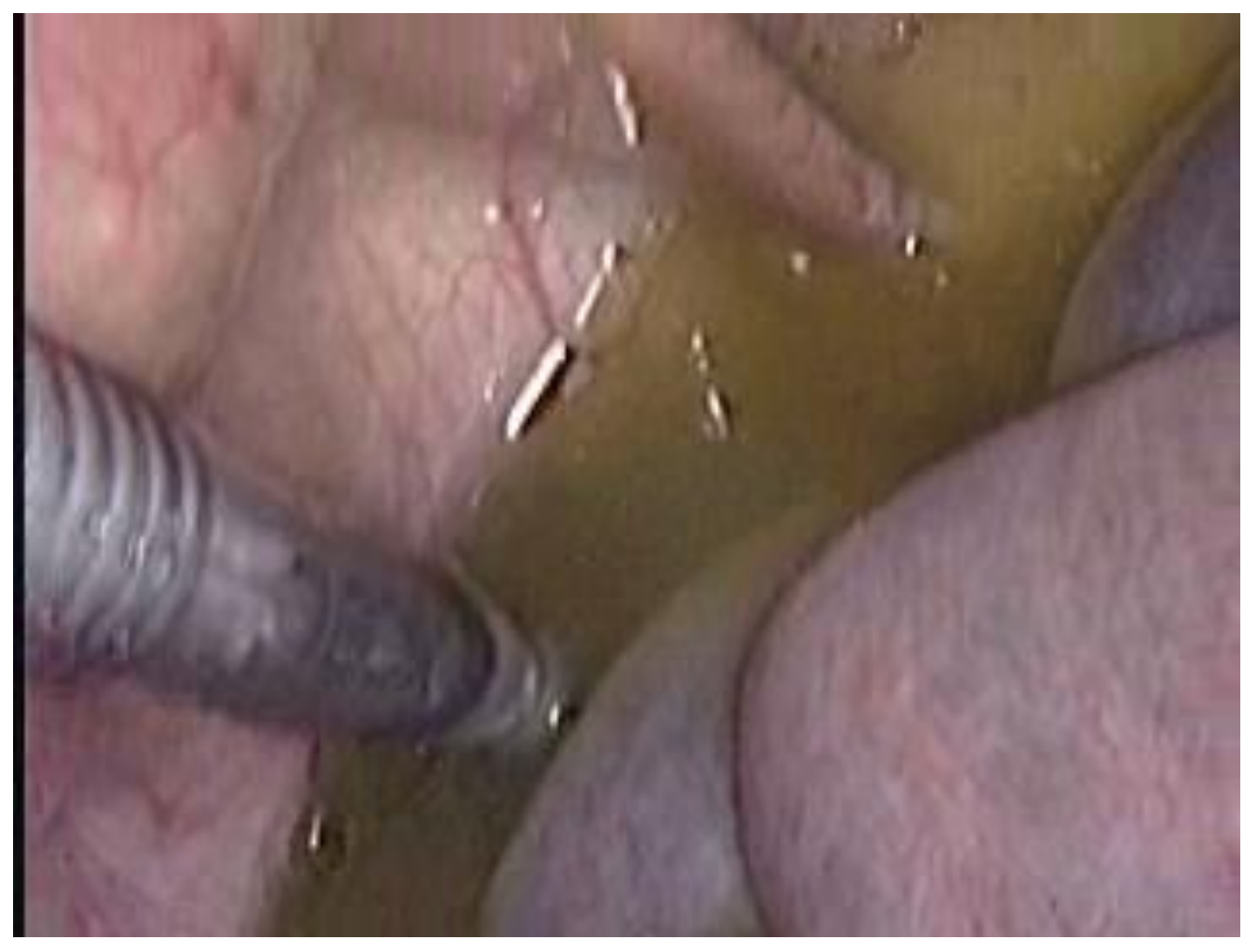

Figure 7: Straw coloured pelvic fluid

\subsection{Omentum:}

Forty one specimens $(51 \%)$ were taken from omentum and sent for histological examination; 15 of them were positive (37\%) for tuberculosis and 26 were negative (63\%). Amongst the 15 positive patients 12 had caseating granuloma $(80 \%)$ and three had non-caseating granuloma (20\%), and only five amongst all of them (33\%) had AFB positive. Amongst the 26 histology negative patients, 12 had nonspecific chronic inflammation, one had acute inflammation (the patient had bowel perforation), three had diagnosis other than tuberculosis (3adenocarcinoma), and ten had no pathology found. 
The sensitivity and specificity of omentum histology are $61 \%(95 \% \mathrm{Cl} 39-80 \%)$ and $94 \%(95 \% \mathrm{Cl} 71-100 \%)$ respectively. The positive predictive value and negative predictive value of omentum are $93 \%(95 \% \mathrm{Cl} 66-100 \%)$ and $65 \%$ $(95 \% \mathrm{Cl} 44-82 \%)$ respectively. Figure 8 is showing taking biopsy from the omentum.

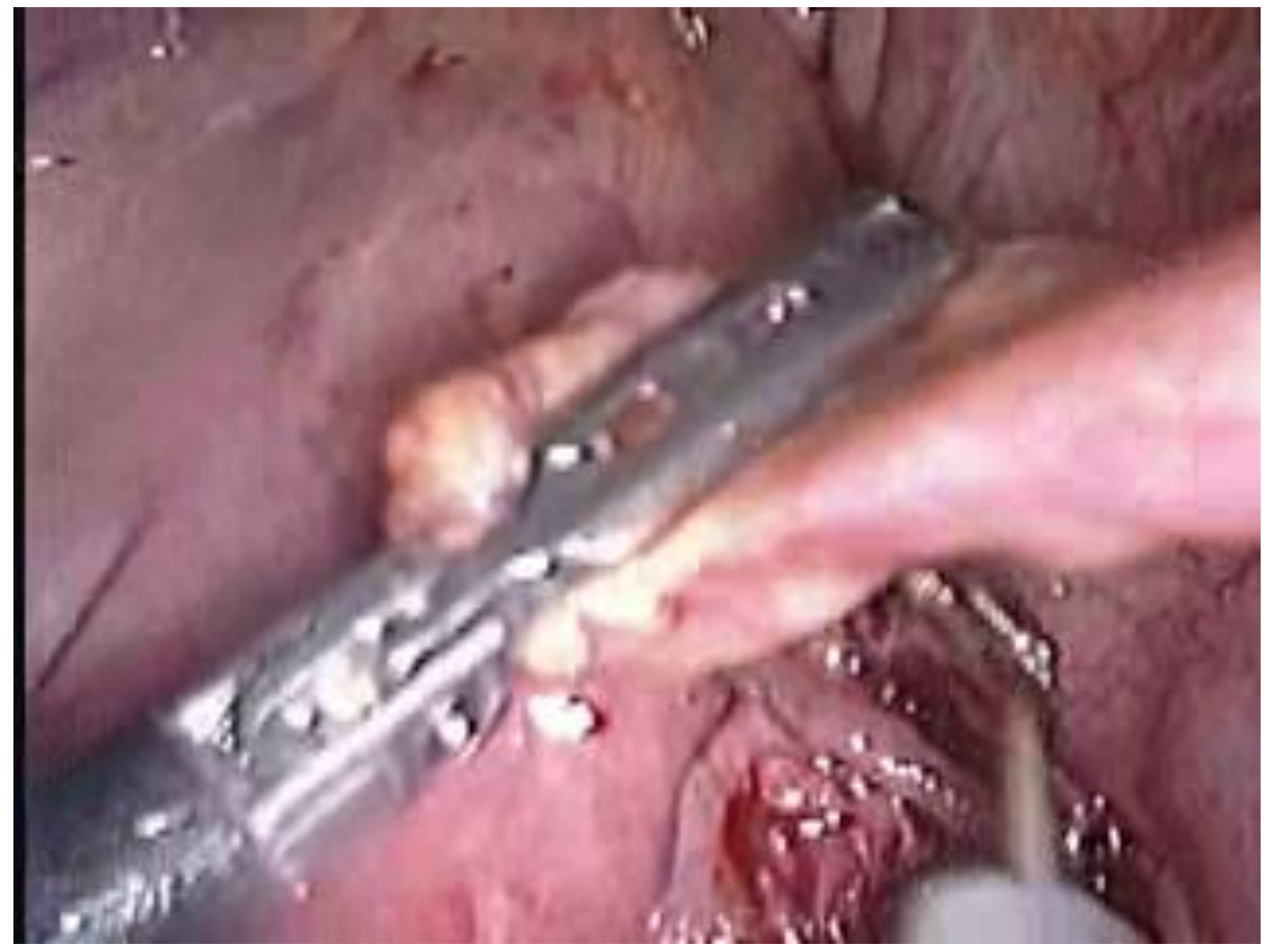

Figure 8: Taking biopsy from the omentum. 


\subsection{Tubercles:}

Peritoneal/ omental deposits (tubercles) were present and sent for histological examination in 20 patients (25\%) and there was no deposit in the other 60 patients $(75 \%) ; 16$ of them were positive $(80 \%)$ for tuberculosis and four $(20 \%)$ were negative. Amongst the 16 positive patients 13 had caseating granuloma (81\%) and three had non-caseating granuloma (19\%), and only six amongst all of

them (37\%) had AFB positive. Amongst the four negative patients, two had diagnosis other than tuberculosis (2-adenocarcinoma) and two had nonspecific chronic inflammation.

No specimen was taken from one patient. The sensitivity and specificity of tubercles are $31 \%(95 \% \mathrm{Cl} 19-45 \%)$ and $86 \%(95 \% \mathrm{Cl} 66-95 \%)$ respectively. The positive predictive value and negative predictive value of tubercles are $80 \%$ (95\% Cl 56-93\%) and 40\% (95\% Cl 28-53\%) respectively. Figure 9 is showing multiple tubercles over the lleo-caecal region. 


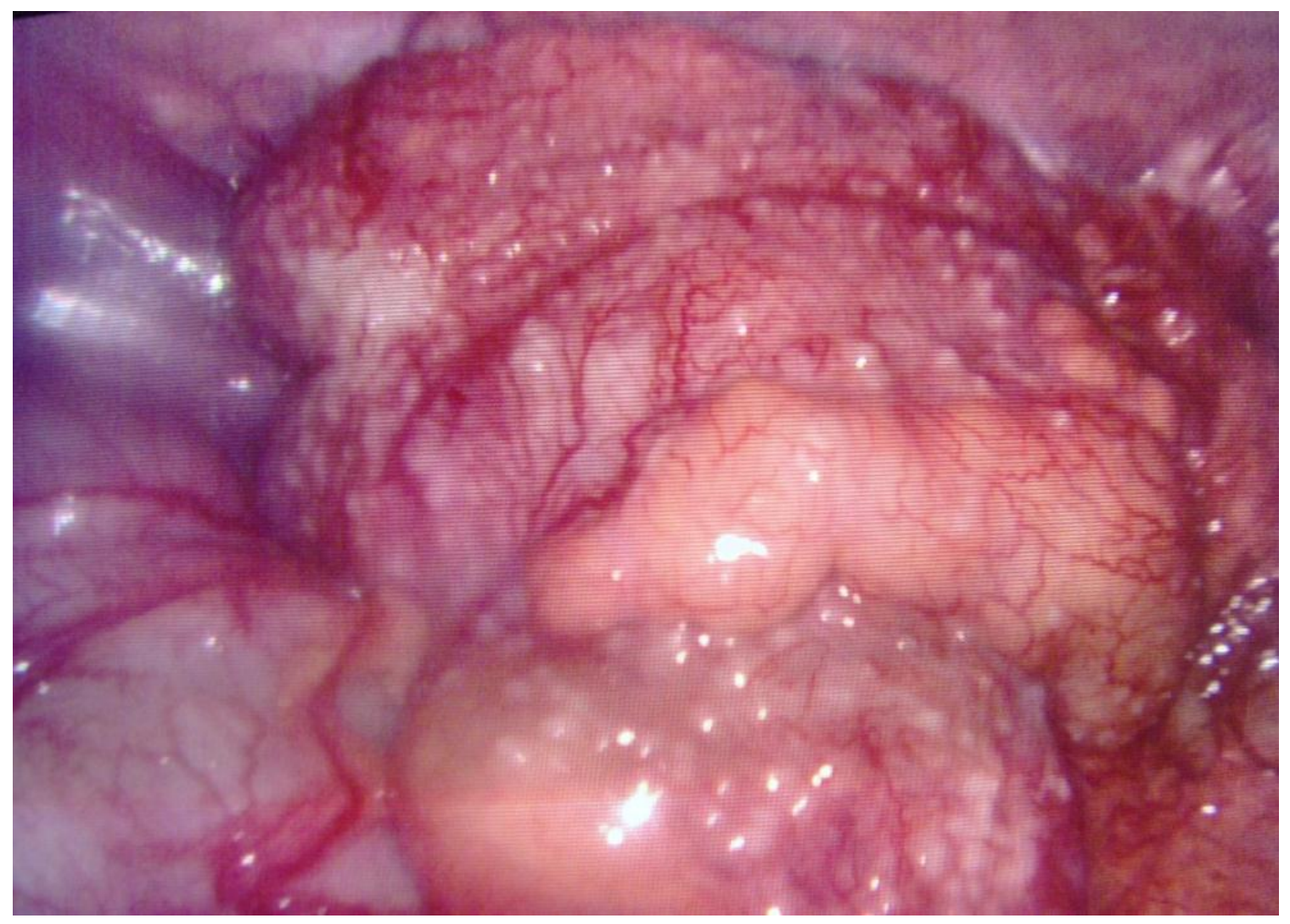

Figure 9: Multiple tubercles over the lleo-caecal region

\subsection{Peritoneum in the absence of tubercles:}

Twenty five specimens (31\%) were taken from peritoneum and sent for histological examination; nine of them were positive (36\%) for tuberculosis and 16 were negative (64\%). Amongst the nine positive patients six had caseating granuloma (67\%) and three had non-caseating granuloma (33\%), and only one (11\%) amongst all of them had AFB positive. Amongst the 16 negative patients, three had nonspecific chronic inflammation, one had acute inflammation and 12 had no pathology found. 
The sensitivity and specificity of peritoneal tissue histology are $47 \%(95 \% \mathrm{Cl} 22$ $73 \%)$ and $80 \%(95 \% \mathrm{Cl} 44-96 \%)$ respectively. The positive predictive value and negative predictive value of peritoneum are $78 \%(95 \% \mathrm{Cl} 40-96 \%)$ and $50 \%$ (95\% Cl 26- 74\%) respectively.

\subsection{Abdominal/ retroperitoneal mass:}

Intra-abdominal or retroperitoneal masses were present and biopsied from 17 patients (21\%); histology of masses were positive for tuberculosis in $14(82 \%)$ and negative in three patients (18\%). Amongst the 14 positive patients 12 had caseating granuloma (86\%) and two had non-caseating granuloma (14\%), and eight amongst all of them (57\%) had AFB positive.

No specimen was taken from one patient. The sensitivity and specificity of masses are $27 \%(95 \% \mathrm{Cl} 16-41 \%)$ and $89 \%(95 \% \mathrm{Cl} 71-97 \%)$ respectively. The positive predictive value and negative predictive value masses are $82 \%(95 \% \mathrm{Cl}$ 56- $95 \%)$ and $40 \%(95 \% \mathrm{Cl} 28-53 \%)$ respectively.

\subsection{Appendix:}

Five appendicectomies (6\%) were done along with other tissues for histology and all of them were negative for tuberculosis. Three of them showed features of acute appendicitis with no other tissues positive for tuberculosis in these three patients, and final diagnosis was acute appendicitis. In one patient appendix specimen showed acute inflammation, but positive histology for tuberculosis in 
lymph node. The other appendix specimen was normal, but positive histology for tuberculosis in lymph node.

\subsection{Liver biopsy:}

Three liver biopsies (4\%) were done during laparoscopy. One was positive for tuberculosis showing non-caseating granuloma and also was positive for tuberculosis in other tissues, one showed features of cholestasis but was negative for tuberculosis in other tissues, and the other one was normal.

\subsection{Cholecystectomy:}

Four cholecystectomies (5\%) were done along with other tissue biopsies. All four gall bladder specimens showed features of acute cholecystitis with cholelithiasis, but no evidence of tuberculosis. All of these patients had positive histology for tuberculosis in other tissues. All these patients presented with biliary colic along with constitutional symptoms. U/S examination confirmed the presence of gall stones and they were planned for cholecystectomy and diagnostic laparoscopy at the same time.

During laparoscopy a total of 217 specimens were collected from 80 patients for histological and microbiological examinations and no specimen was taken from one patient. Table 5 is showing the results of laparoscopic specimens. 
Table 5: The results of laparoscopic specimens.

\begin{tabular}{|l|c|c|c|c|c|c|c|}
\hline Specimen & $\begin{array}{c}\text { Number } \\
(\%)\end{array}$ & $\begin{array}{c}\text { Numbers } \\
\text { positive }\end{array}$ & $\begin{array}{c}\text { Positive } \\
\text { (\%) }\end{array}$ & $\begin{array}{c}\text { Sensitivity } \\
\text { (\%) }\end{array}$ & $\begin{array}{c}\text { Specificity } \\
\text { (\%) }\end{array}$ & $\begin{array}{c}\text { PPV } \\
\text { (\%) }\end{array}$ \\
\hline Tubercles & $20(25)$ & 16 & 80 & 31 & 86 & 80 & 40 \\
\hline Lymph nodes & $56(70)$ & 43 & 77 & 83 & 54 & 77 & 63 \\
\hline Omentum & $41(51)$ & 15 & 37 & 61 & 94 & 93 & 65 \\
\hline Peritoneum & $25(31)$ & 9 & 36 & 47 & 80 & 78 & 50 \\
\hline Ascitic fluid & $46(58)$ & 16 & 35 & 46 & 83 & 81 & 50 \\
\hline Liver biopsy & $3(4)$ & 1 & 33 & N/A & N/A & N/A & N/A \\
\hline Appendix & $5(6)$ & Nil & Nil & N/A & N/A & N/A & N/A \\
\hline Gall bladder & $4(5)$ & Nil & Nil & N/A & N/A & N/A & N/A \\
\hline Total & $\mathbf{2 1 7}$ & $\mathbf{1 1 4}$ & $\mathbf{5 3}$ & & & & \\
\hline
\end{tabular}

$\mathrm{PPV}=$ Positive Predictive Value, $\mathrm{NPV}=$ Negative Predictive Value

*Percent rounded to the nearest integer

The sensitivity, specificity, PPV and NPV calculations were based on the number who had the sample taken, are not on the total number (81) undergoing laparoscopy.

114 specimens taken from different sites were positive for tuberculosis either on histological or microbiological examinations.

Table 6 (in the appendix) showing the breakdown of alternate diagnosis and tuberculosis positive patients in laparoscopic specimens. 
Total laparoscopic specimens (217) - ascitic fluid specimens (46) $=$ total specimens (171) sent for histological examination. Table 7 shows the histology results of 171 specimens. 
Table 7: Histology results of 171 specimens.

\begin{tabular}{|c|c|c|c|c|c|c|c|c|c|}
\hline \multirow[t]{2}{*}{ Specimens } & \multicolumn{2}{|c|}{ Numbers } & \multirow{2}{*}{$\begin{array}{c}\text { Caseating } \\
\text { granuloma } \\
\text { No (\%) }\end{array}$} & \multirow{2}{*}{$\begin{array}{c}\text { Non-caseating } \\
\text { granuloma } \\
\text { No (\%) }\end{array}$} & \multirow{2}{*}{$\begin{array}{c}\text { AFB } \\
\text { positive } \\
\text { No (\%) }\end{array}$} & \multirow{2}{*}{$\begin{array}{c}\text { Chronic } \\
\text { inflammation } \\
\text { No }(\%)\end{array}$} & \multirow{2}{*}{$\begin{array}{c}\text { Acute } \\
\text { inflammation } \\
\text { No (\%) }\end{array}$} & \multirow{2}{*}{$\begin{array}{c}\text { No pathology } \\
\text { No (\%) }\end{array}$} & \multirow{2}{*}{$\begin{array}{c}\text { Other } \\
\text { diagnosis } \\
\text { No (\%) }\end{array}$} \\
\hline & Taken & + & & & & & & & \\
\hline Lymph node & 56 & 43 & $35(63)$ & $8(14)$ & $24(43)$ & $10(18)$ & --- & $1(2)$ & $2(4)$ \\
\hline Omentum & 41 & 15 & $12(29)$ & $3(7)$ & $5(12)$ & $12(29)$ & $1(2)$ & $10(24)$ & $3(7)$ \\
\hline Peritoneum & 25 & 9 & $6(24)$ & $3(12)$ & $1(4)$ & $3(12)$ & $1(4)$ & $12(48)$ & --- \\
\hline Tubercles & 20 & 16 & $13(65)$ & $3(15)$ & $6(30)$ & $2(10)$ & --- & --- & $2(10)$ \\
\hline Mass & 17 & 14 & $12(71)$ & $2(12)$ & $8(47)$ & $2(12)$ & --- & --- & $1(6)$ \\
\hline Appendix & 5 & --- & --- & -- & --- & --- & $4(80)$ & $1(20)$ & --- \\
\hline Gall bladder & 4 & --- & --- & --- & --- & --- & $4(100)$ & --- & --- \\
\hline Liver tissue & 3 & 1 & --- & $1(33)$ & --- & --- & --- & $1(33)$ & $1(33)$ \\
\hline Total & 171 & 98 & $78(46)$ & $20(12)$ & $44(26)$ & $29(17)$ & $10(6)$ & $25(15)$ & $9(5)$ \\
\hline
\end{tabular}

${ }^{*}$ Percent rounded to the nearest integer

The percentage calculations are based on the percentage of overall number of specimens taken. 
Table 8 shows the overall histology results of 171 specimens.

Table 8: Overall histology results of 171 specimens.

\begin{tabular}{|l|l|c|c|}
\hline Tuberculosis & Findings & Number & $\%$ \\
\hline Positive: 98 & Caseating granuloma & 78 & 46 \\
\cline { 2 - 4 } AFB+: 44 (45\%) & Non caseating granuloma & 20 & 12 \\
\hline Negative: 73 & Chronic inflammation & 29 & 17 \\
\cline { 2 - 4 } & Acute inflammation & 10 & 6 \\
\cline { 2 - 4 } & No pathology & 25 & 14 \\
\cline { 2 - 4 } & Other diagnosis & 9 & 5 \\
\hline Total & & $\mathbf{1 7 1}$ & $\mathbf{1 0 0}$ \\
\hline
\end{tabular}

*Percent rounded to the nearest integer

Due to the overlapping of different results from the various specimens from the same patient, the overall result was: 15 had non-specific chronic inflammations, one had no pathology found. Ten patients with acute inflammations: four had acute appendicitis (no tuberculosis in any specimen), four had acute cholecystitis (all four had tuberculosis in other specimens), one had non-specific bowel perforation and one had tuberculosis in other tissue. 
Amongst the 81 patients, 20 had non-specific chronic inflammation and 15 had no pathology in different specimens. The rest of the patients had positive tuberculosis in various specimens except one patient, from whom no specimen was taken. Due to the overlapping of various specimens from the same patient, a total of 15 patients had non-specific chronic inflammation and one patient had no pathology found. The patients in the table 9 (in the appendix) with shaded areas (16) had neither tuberculosis nor any other diagnosis.

Table 10 (in the appendix) is showing the breakdown of all tuberculosis positive results from histology, blood and ascitic fluid culture.

Fifty two patients had histology positive for tuberculosis. Three patients had histology negative but blood/ ascitic fluid culture positive, showed in shaded areas in the table 10 (in the appendix). Total $(52+3) 55$ patients had positive tuberculosis findings. Twenty six patients were negative for tuberculosis but nine of them had alternate diagnosis and no specimen was taken from one patient. The histology, blood culture and ascitic fluid culture were all negative for tuberculosis in 16 patients, but it showed non-specific chronic inflammation in 15 and no pathology was found in one patient. The histology, blood culture and ascitic fluid culture were all positive in six patients. Sensitivity and specificity of these couldn't be established because the numbers of patients are not the same in all the group of tests. 
Total 217 specimens were taken from 81 patients for histological and microbiological examinations. Figure 10 is showing the breakdown of tuberculosis positive results according to the specimens.

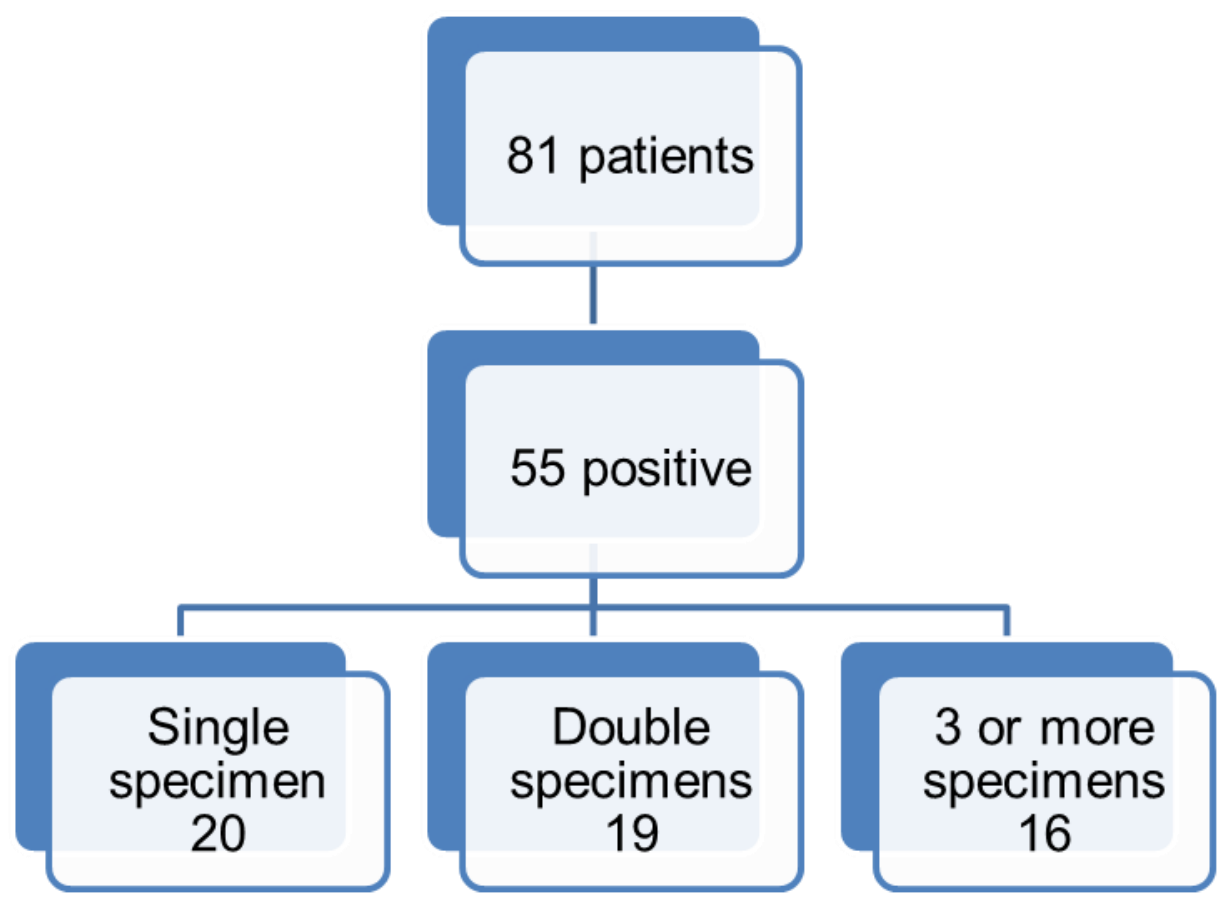

Figure 10: Tuberculosis positive result according to the number of specimens positive

Total nine patients $(11 \%)$ had diagnosis other than tuberculosis. Of these nine patients; three had adenocarcinoma, three appendicitis, one lymphoma, one portal hypertension and one non-specific bowel perforation. Three patients had adenocarcinoma from multiple specimens e.g. lymph node, omentum, tubercle and mass; all other diagnosis were made from single specimen (lymph node, 
appendix, liver and bowel tissue). Six of these nine patients were HIV positive (67\%), two were unknown (22\%) and one was negative (11\%). Despite the negative result for tuberculosis; four had high ESR (44\%), three had high CRP (33\%) and six had suggestive tuberculosis findings in CT scan (67\%).

Twenty six patients (32\%) were negative for tuberculosis (15 non-specific chronic inflammation, 1 no pathology, 9 other diagnosis, 1 no specimen was taken). Table 11 (in the appendix) is showing the breakdown of these 26 patients. Of these 26 tuberculosis negative patients, 18 were HIV positive (69\%), three were unknown (12\%), and five were negative (19\%). Despite the negative result for tuberculosis; 10 had high ESR (38\%), eight had high CRP (31\%) and 17 had suggestive tuberculosis findings in CT scan (65\%).

Sixteen patients $(20 \%)$ were negative for tuberculosis with no other diagnosis (15 with non-specific chronic inflammation and one with no pathology) and these 16 patients were considered true negatives for tuberculosis. Table 12 (in the appendix) is showing the breakdown of 16 patients who are tuberculosis negative with no other diagnosis. Eleven of these 16 patients were HIV positive $(69 \%)$, one was unknown (6\%) and four were negative (25\%). Despite the negative result for tuberculosis; five had high ESR (31\%), four had high CRP (25\%) and 11 had suggestive tuberculosis findings in CT scan (69\%). 
Figure 11 illustrates the laparoscopic results in relation to diagnosis and HIV status

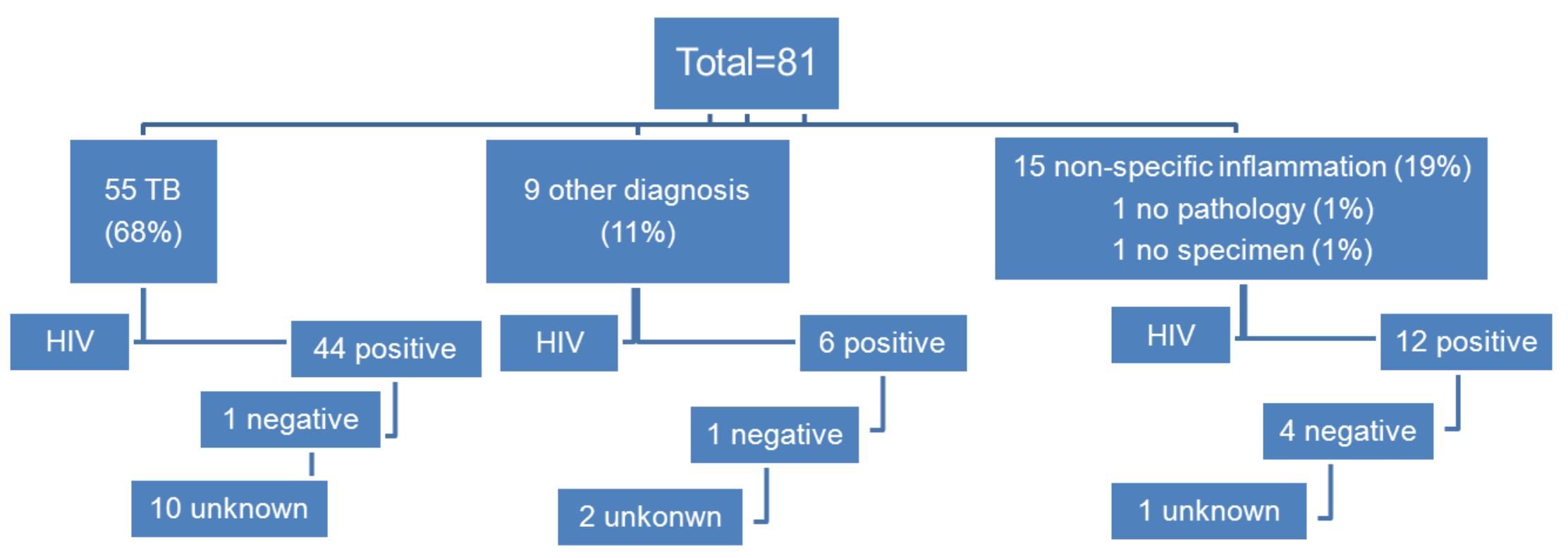

Figure 11: Laparoscopic results in relation to HIV status. 


\section{Complications}

A total nine patients $(11 \%)$ had conversion to laparotomies for various reasons mentioned in table 13; two of them had laparotomies on a later occasion due to peritonitis, not during laparoscopy. There was no death related to conversion to laparotomy. Table 13 is showing the breakdown of nine patients who had conversion to laparotomies.

Table 13: Conversion to laparotomy.

\begin{tabular}{|c|c|c|c|c|c|}
\hline No. & Age & Sex & Reason for conversion & Histology & HIV \\
\hline 1 & 23 & $\mathrm{~F}$ & Technical: due to laparoscope & TB & Unknown \\
\hline 2 & 32 & $\mathrm{M}$ & Bowel perforation during access & TB & + \\
\hline 3 & 32 & $\mathrm{~F}$ & Technical: due to laparoscope & TB & + \\
\hline 4 & 25 & $\mathrm{M}$ & Bowel perforation during access & Adeno-carcinoma & + \\
\hline 5 & 18 & $\mathrm{M}$ & Adhesions & Non-specific bowel & + \\
\hline 6 & 35 & $\mathrm{~F}$ & perforation & \\
\hline 7 & 27 & $\mathrm{M}$ & Bleeding & TB & Unknown \\
\hline 8 & 34 & $\mathrm{~F}$ & Adhesions & TB & Unknown \\
\hline 9 & 31 & $\mathrm{M}$ & Bleeding & TB specimen & + \\
\hline
\end{tabular}




\section{Deaths}

A total nine patients (11\%) died during the two months follow up post surgery. All of these patients were HIV positive. Four patients died in our hospital. The diagnoses of these four were adenocarcinoma (1), lymphoma (1), non-specific bowel perforation (1), chronic inflammation (1), and the cause of death were advanced cancer (2), abdominal sepsis (1), and advanced AIDS (1). Five patients died in the local hospital after discharged from us and the cause of death could not be established. Two of them had TB, two appendicitis and one chronic inflammation. 


\section{Follow up}

Follow up of our patients was very poor. We followed the patient up to two months post laparoscopy. Figure 12 is showing the follow up patients.

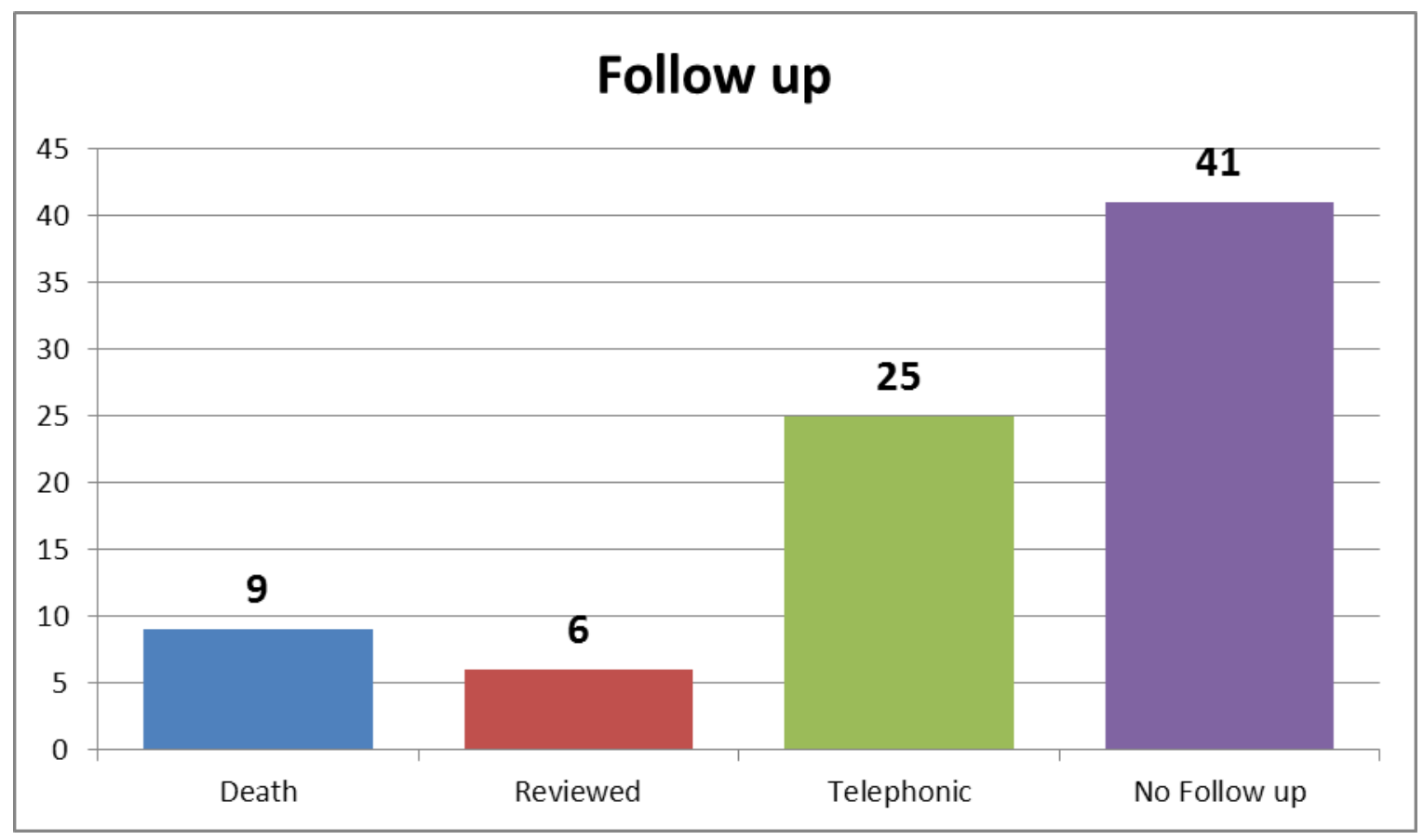

Figure 12: Follow up of 81 patients.

Nine patients died (11\%) during that two months period, the remaining 72 patients $(89 \%)$ were followed up. Only six patients (8\%) came for review, all of them had positive diagnosis of tuberculosis and all of them improved symptomatically with anti TB medications. Repeat abdominal U/S, Hb\%, CRP were done and body weight were taken in all six patients and a trend of improvement was noted in all patients. 
In the remaining patients telephonic follow up was attempted. Only 25 patients (35\%) could be contacted. In the rest there was no response from the contact numbers they provided. Of these 25 patients, 15 had positive tuberculosis diagnosis, seven had negative diagnosis and three had other diagnosis (portal hypertension, adenocarcinoma and appendicitis). All 25 patients reported resolution of their symptoms. 


\section{Group B: Laparotomy}

During the study period another forty nine patients were admitted with acute abdominal condition and had emergency laparotomy for various indications. All 49 patients had positive histological diagnosis of abdominal tuberculosis. Twenty five were male, 24 female and the mean age was 32 years $(95 \% \mathrm{Cl} 29-36$ years). Thirty nine were HIV positive (80\%) and HIV status of the others were unknown or negative.

Only six patients had a prior CT abdomen. Twelve patients presented with obstruction (24\%), nine with perforation (18\%) and 28 with peritonitis without free air (57\%). Intra-operative findings were: frozen abdomen in 10 (20\%), bowel perforations in 13 (27), enlarged lymph nodes and ileo-caecal mass in $19(3 \%)$ and obstructed small bowel in seven patients (14\%). Eleven patients (22\%) had small bowel resection and eight patients (16\%) had right hemicolectomies. Eighteen patients (37\%) ended up with stomas (16 ileostomies and two colostomies) and only two had primary anastomoses. Fourteen patients (29\%) had relaparotomies and 17 patients (35\%) were admitted to ICU. Twenty three patients $(47 \%)$ required blood transfusion and 15 patients $(31 \%)$ required total parenteral nutrition. Three patients developed enterocutaneous fistula and five patients were discharged with ventral hernia. Nineteen patients died $(39 \%)$ of whom only six had bowel resection and ten of them were admitted to ICU. 


\section{Group C: No surgical intervention}

Another 60 patients were also evaluated for the diagnosis of abdominal tuberculosis on the basis of clinical features and radiological findings. Thirty five were male, 25 female and the mean age was 32 years (95\% Cl $30-34$ years). All of them were HIV positive. Twenty six patients died (43\%) in the hospital during the evaluation period before the diagnostic laparoscopy, and the rest (57\%) were unfit for anaesthetic for diagnostic laparoscopy. This group of patients was not further followed up by us. 


\section{Discussion}

It has been established that the incidence of all tuberculosis, including abdominal tuberculosis is rising in South Africa and the diagnosis is still difficult. This study was conducted at Edendale Hospital, Pietermaritzburg a 860-bed district and regional hospital in a semi-urban area in the province of Kwa-Zulu Natal, South Africa which serves a mainly ethnic African population of 1.6 million, and a referral centre for tuberculosis patients from local clinics. The number of patients with abdominal tuberculosis has been increasing. Clarke et al $^{17}$ published a series of 67 patients accumulated over 18 months from July 2003 to January 2005 at Addington hospital in the same province with a similar catchment population, whereas we had 190 patients over 30 months period from January 2008 to June 2010.

It is therefore essential that the clinicians consider tuberculosis in the evaluation of abdominal pain, ascites, obstruction, or peritonitis if diagnosis at autopsy is to be avoided. ${ }^{67}$ The mortality of tuberculosis peritonitis is $47-49 \%$ if untreated, although it can be less than $5 \%$ with treatment, several studies report a mortality of up to $60 \%$ mainly because of delayed or missed diagnosis. ${ }^{29,66}$ To avoid any delay or missed diagnosis we wished to look at the utility of diagnostic laparoscopy in abdominal tuberculosis. Although the concept of diagnostic laparoscopy in tuberculosis is not new, ${ }^{65,66}$ the patients profile, clinical presentations, equipment and method used at present are completely different from that of the past. Historically peritoneal biopsy or peritoneoscopy used to be 
done under local anaesthetics with a limited view of the abdominal cavity and higher complication rates, whereas at present laparoscopy is performed under general anaesthetics with better intra-operative views and less complication rates. $^{68,77}$ The clinical profile of our patients are very different from the patients in the past. The majority of our patients are HIV positive (77\%) with dry (plastic type) abdominal tuberculosis, in comparison the patients from earlier time periods before the HIV epidemic set in the hospital prevalence was considerabley lower . The majority had ascites, no HIV disease, but with other risk factors for the development of tuberculosis. ${ }^{19,29,68,78,79}$

\subsection{Clinical characteristics:}

The male female ratio is highly variable from equal in the series reported by Ramesh et $\mathrm{al}^{80}$ to a male predominance reported in two studies ${ }^{56,79}$ and to a marked female predominance in the study of Nafeh et al. ${ }^{78}$ In our series the male: female ratio of 1:3. The reasons for these variations are unclear.

Total 55 patients had positive diagnosis of tuberculosis (68\%) and most of these diagnoses were made from lymph nodes histology (77\%). HIV disease is common in our cohort, $77 \%$ of the 81 patients were HIV positive and amongst the 55 tuberculosis positive patients $80 \%$ were HIV positive. Our series is biased due to suspected HIV being the entry criteria, however it is interesting to note that most of the reported series of abdominal tuberculosis in the literature are either HIV negative or not mentioned. ${ }^{78,79,80}$ Reports from Zambia ${ }^{81}$, Nigeria ${ }^{82}$ and 
India ${ }^{15}$ indicate a lesser abdominal tuberculosis and HIV co-infection rate than South Africa. ${ }^{11}$ Ghiya et al ${ }^{15}$ reported $49 \%$ of HIV positive patients are co-infected with tuberculosis in Gujarat, India and $50 \%$ of all tuberculosis were abdominal tuberculosis. Sinkala et $\mathrm{al}^{81}$ reported $73 \%$ of medical inpatients in Zambia were HIV positive and $71 \%$ of them had definite or probable abdominal tuberculosis. lliyasu et $\mathrm{al}^{82}$ reported $10 \%$ of HIV positive patients are coinfected with tuberculosis in Nigeria and $14 \%$ of all tuberculosis were abdominal. Although in South Africa approximately $80 \%$ of incident tuberculosis cases are HIV seropositive, the true incidence of abdominal tuberculosis is not well established. ${ }^{11}$ Clarke et al ${ }^{17}$ reported a $34 \%$ hospital prevalence of HIV in their patients with abdominal tuberculosis however $10 \%$ were HIV negative and more than $50 \%$ of their HIV status was unknown.

The mean duration of symptoms of our patients was 41 days which is similar to other series. ${ }^{79}$ The most common presentation was abdominal pain $(100 \%)$, followed by weight loss (85\%) and night sweats (75\%) which is more or less similar in other series including the gastrointestinal symptoms. ${ }^{78,79}$ Generalized lymphadenopathy was much more common in our series (64\%) than the others $(11 \%$ and $39 \%)$ most probably due to high prevalence of HIV infection. ${ }^{78,81}$ Fever was less common in our series (24\%) than in others (46\% to $92 \%) .{ }^{78,79,80}$ Ascites was present in $94-95 \%$ of patients in some of the series in the past ${ }^{18,78}$ and in 61$65 \%$ of patients in some of the recent series, ${ }^{56,83}$ in comparison only $2 \%$ of our patients had clinical evidence of ascites. Many patients in other series had 
associated risk factors e.g. liver cirrhosis, end stage renal diseases requiring peritoneal dialysis, diabetes mellitus, prolonged steroid therapy etc..$^{29,79}$

\subsection{Preoperative investigations:}

Normocytic normochromic anaemia was present in $91 \%$ of patients which was similar to the rate found by Nafeh et al. ${ }^{78}$ Nineteen patients $(23 \%)$ had perioperative blood transfusions.

Variations in the white cell counts are difficult to interpret in view of the high HIV hospital prevalence and the effect HIV infection has on the lymphocyte count. We found that leucocytosis was more common than leucopenia (22\% vs $15 \%$ ) which was similar to that reported by Manohar et $\mathrm{al}^{18}$ in the pre HIV era in Durban.

\subsection{Imaging:}

The chest radiographs of only ten patients (12.\%) were suggestive of tuberculosis with pulmonary infiltrates, though sputum cultures were negative in all 10 patients; a percentage half of that reported in other series. ${ }^{78,80}$

$\mathrm{U} / \mathrm{S}$ abdomen was suggestive of tuberculosis in all patients, but histology/culture results were positive in 55 patients. Though the sensitivity of CT scan was high, the specificity was very low in our series. In the series presented by Bolukbas et $\mathrm{al}^{84}$, the evidence of abdominal tuberculosis e.g. ascites, gut wall thickness, lymphadenopathy, abscess, and organomegally were detected in $82 \%$ of 
patients, though all their patients were HIV negative. Caseating lymph nodes with hypodense centres and peripheral rim enhancement along with calcification are highly suggestive of tuberculosis. ${ }^{43}$

\subsection{ESR/CRP:}

Histology/culture for tuberculosis was positive in $69 \%$ of patients with high ESR. Three patients had normal ESR, one of whom had tuberculosis. Seventy five percent of patients with ESR more than 100 had tuberculosis, and the rest had either no tuberculosis or other diagnosis. In patients with ESR less than 100, $54 \%$ of them had tuberculosis. Ten patients with high ESR (31\%) had no tuberculosis. Four patients with high ESR (12\%) had diagnosis other than tuberculosis. ESR is an acute phase reactant and the elevated level is neither specific nor absolutely accurate test and it is commonly used as an indicator of certain underlying diseases e.g. infections, inflammations and malignancies, and to monitor the progress and response to therapy. It has been suggested that the degree of sensitivity and / or specificity of the ESR becomes more acceptable at ESR values of $100 \mathrm{~mm} /$ hour and higher with high correlation with bacterial infection, though still not a useful diagnostic test especially in the presence of HIV infection. ${ }^{85,86}$ Though Ukpe et al $^{87}$ suggested that active tuberculosis is associated mostly with very high ESR values $(\geq 100 \mathrm{~mm} / \mathrm{h}$ ) irrespective of HIV status, Al-Marri et $\mathrm{al}^{88}$ suggested that the ESR value is likely to be of little or no diagnostic utility in the diagnosis of tuberculosis especially in the children. 
Much of the data on CRP as an adjunct in tuberculosis diagnosis relates to pulmonary tuberculosis. Though Wilson et $\mathrm{al}^{89}$ described as in high HIV prevalence settings, a normal CRP could be a useful test in combination with clinical evaluation to rule out tuberculosis; three of our patients with normal CRP (75\%) had positive histology and eight patients with high CRP (40\%) had negative histology. Three patients with high CRP (15\%) had diagnosis other than tuberculosis (malignancies, appendicitis). One patient with high CRP $(24 \mathrm{mg} / \mathrm{L})$ no specimen was taken. The role of CRP in tuberculosis and HIV co-infection is still not well defined. Sage et $\mathrm{al}^{90}$ reported that all patients with pneumocystis jirovecii pneumonia, bacterial pneumonia or pulmonary tuberculosis may not have an elevated CRP, which suggests that an active respiratory infection cannot be excluded among HIV infected patients with respiratory symptoms on the basis of a normal CRP value. Noursadeghi et al ${ }^{91}$ reported that in HIV infected patients without intercurrent infection, CRP values may be higher than in the general population possibly reflecting a sustained acute phase response as a consequence of HIV infection per se. HIV - seropositive patients with community acquired pneumonia have significantly higher CRP levels than those with pulmonary tuberculosis despite similar clinical and radiological appearances. ${ }^{92}$ Chaudhary et $\mathrm{al}^{93}$ reported that symptomatic mild inflammatory disease or HIV infection commonly increases CRP concentration mildly and CRP was negatively correlated with CD4 counts in HIV positive patients. 


\subsection{Blood culture:}

Blood culture was positive for tuberculosis only in $25 \%$ of patients. Only in two patients blood culture was positive but histology was negative. Da Bouza et al ${ }^{94}$ showed that blood culture was positive for tuberculosis in $50 \%$ of patients with disseminated tuberculosis in AIDS patients, though the sample size was very small. Whereas Wilson et $\mathrm{al}^{8}$ showed blood culture was positive for tuberculosis in $28 \%$ of patients (130 patients), which is similar to our series.

\subsection{PCR:}

Though the PCR systems developed so far have shown good levels of sensitivity $(90-100 \%)$ in AFB smear-positive samples, ${ }^{95}$ and due to the lack of facilities and resources we did not perform any PCR test. The newer MTB/RIF test can detect tuberculosis in $72 \%$ of smear-negative and $98 \%$ of smear-positive pulmonary tuberculosis, and can identify $97 \%$ of rifampicin resistant bacteria in less than two hours. ${ }^{96}$ The biggest limitation of this test is the high cost of the equipment, test cartridges and the maintenance of the machines; the cost of each disposable test-cartridge is comparable with the per capita annual health expenditure in the

countries with the highest tuberculosis burdens. ${ }^{97}$ The role of MTB/RIF test in diagnosing abdominal tuberculosis is unknown. 


\subsection{Endoscopy:}

Investigations to assess luminal tuberculosis were performed infrequently, with approximately $20 \%$ having either an upper GI endoscopy or a colonoscopy and less than three percent having a contrast study and none of these investigations suggested or proved tuberculosis. Gastrointestinal tuberculosis was reported to be $37 \%-50 \%$ of abdominal tuberculosis in the 70 's and 80 's. ${ }^{16,25}$ There was no patient with documented luminal intestinal tuberculosis in our series and most of our patients presented with abdominal lymphadenopathy (70\%), which was similar to the series by Clarke et al. $^{17}$ Ramesh et $\mathrm{al}^{80}$ reported that $44 \%$ of abdominal tuberculosis in their series was intestinal tuberculosis, though HIV coinfection was not mentioned in the series. Most of our patients were African ethnic origin in whom inflammatory bowel disease, especially Crohn's disease is a very unusual diagnosis. That's why only a few of our patients went for colonoscopy, whereas differentiating Crohn's disease from tuberculosis is a major issue in the Indian, Colored and White populations. So in areas of South Africa where these ethno social groups cluster, colonoscopy plays a major role in distinguishing Crohn's disease from tuberculosis. ${ }^{51}$

\subsection{Laparoscopy:}

Previously mesenteric lymphadenopathy was not the most common findings in abdominal tuberculosis. Nafeh et $\mathrm{al}^{78}$ did not have any patients with mesenteric lymphadenopathy, while Marks et $\mathrm{al}^{98}$ had $10 \%$ and Clarke et $\mathrm{al}^{17}$ had $23 \%$ of their patients with mesenteric lymphadenopathy. In contrast mesenteric 
lymphadenopathy was the most common finding in our series and the highest number $(70 \%)$ of our patients had mesenteric lymph nodes biopsied. Mesenteric lymph node was the $3^{\text {rd }}$ highest $(77 \%)$ histology positive and the highest sensitive (83\%) tissue biopsied.

Though only $2 \%$ of our patients had clinical ascites in contrast $90-94 \%$ in the previous series, ${ }^{78,99}$ the second highest numbers of specimens in this series were taken from ascitic fluid found at laparoscopy. The amount of fluid present during laparoscopy varied widely from minimal amount to $200 \mathrm{ml}$, except in two patients where it was about $500 \mathrm{ml}$ and up to $20 \mathrm{ml}$ of specimen was sent for tuberculosis culture from each patient. In our series $35 \%$ of ascitic fluid specimens were positive for tuberculosis culture, in comparison to $23 \%$ in series by Menzies et al $^{19}$ and $16 \%$ in series by Nafeh et al. ${ }^{78}$ Three patients had ascitic fluid culture positive but no other positive histology from any specimen, though two of these patients had positive blood culture as well.

Tubercles were present only in $25 \%$ of our patients, whereas Nafeh et al ${ }^{78}$ had $58 \%$ and Al-Mulhim et $\mathrm{al}^{21}$ had $91 \%$ of their patients with tubercles. Though the number of specimen was small, the $2^{\text {nd }}$ highest positive result was from the tubercles $(80 \%)$.

Intra abdominal/ retro peritoneal mass were present only in $21 \%$ of our patients, but the highest positive result (82\%) was from the masses. Only three patients 
had negative results and amongst them one had adenocarcinoma and the other two had nonspecific chronic inflammations.

Adhesions were present in 18 patients (22\%) in our series which was less than in the series by Nafeh et $\mathrm{al}^{78}(42 \%)$ and Al-Mulhim et $\mathrm{al}^{21}(52 \%)$. Five of the patients with adhesions (6\%) were converted to laparotomy in our series and $16 \%$ of patients were converted to laparotomy due to adhesions in the series by Mimica et al. ${ }^{69}$

The most tuberculosis positive histology specimens are intra / retro peritoneal mass $(82 \%)$, tubercles $(80 \%)$ and lymph nodes $(77 \%)$. There was no positive histology at all for tuberculosis in appendix or gall bladder specimens. The most sensitive specimen is lymph node (83\%) and the most specific specimen is omentum (94\%). The least sensitive and specific specimens are mass (27\%) and peritoneum (80\%) respectively.

Out of 98 tuberculosis positive specimens, 78 of them had caseating granuloma (80\%), 20 had non-caseating granuloma (20\%); with 44 of these specimens being positive for AFB (45\%). Our findings were little different from that of Nafeh et $\mathrm{al}^{78}$, who had caseating granuloma in $34 \%$ and non-caseating granuloma in $56 \%$ cases; though more or less similar to that of Al-Mulhim et $\mathrm{al}^{21}$, who had caseating granuloma in $67 \%$ and non-caseating granuloma in $14 \%$ cases, neither 
of these two studies mentioned about presence of AFB in their specimens and Krishnan et $\mathrm{al}^{71}$ had no AFB present in their ascitic fluid.

\subsection{Diagnosis other than tuberculosis:}

Nine patients had diagnosis other than tuberculosis $(11 \%)$ and these nine patients would have been started on anti-tuberculosis therapy based on clinical and radiological findings, if laparoscopy was not performed. Six of these patients were HIV positive (67\%), ESR was high in 44\%, CRP was high in $33 \%$ and CT

scan was suggestive of tuberculosis in $67 \%$ cases. Menzies et $\mathrm{al}^{66}$ reported $8 \%$ cancers, $43 \%$ liver diseases and $37 \%$ tuberculosis in their series of laparoscopic investigation in patients with ascites.

\subsection{Non-specific chronic inflammations:}

Our main concern is 16 patients (20\%) who are tuberculosis negative and had no other alternative diagnosis. Fifteen of them had non-specific chronic inflammation (18\%) and one had no pathology (1\%). Al-Mulhim et $\mathrm{al}^{21}$ had $19 \%$ non-specific chronic inflammation, whereas Nafeh et $\mathrm{al}^{78}$ had $3 \%$ of their patients with nonspecific chronic inflammation and $7 \%$ with unsatisfactory biopsy.

Eleven of these patients were HIV positive (69\%), high ESR was in $31 \%$, high CRP was in $25 \%$ and CT scan was suggestive of tuberculosis in $69 \%$ cases. Amongst these 16 patients; 12 had lymph node present, two had adhesions and two with absent lymph node. Eight patients with lymph node present were HIV 
positive. It has been seen that most of the HIV positive patients have abdominal lymphadenopathy and it is possible to have missed some diagnosis due to unable to take adequate biopsy due to adhesions. One patient complicated with intra operative bleeding and no specimen was taken from this patient.

All of these 16 patients were started on anti-tuberculosis therapy and two of them died during the two-month follow up period. Seven patients were contacted telephonically and reported to be doing well on anti-tuberculosis therapy. Other seven patients lost in follow up.

\subsection{Perioperative complications/ deaths:}

Nine patients $(11 \%)$ had peri operative complications e.g. bleeding and bowel perforation and had to be converted to laparotomy. Most of these laparotomies are for bleeding (33\%); $22 \%$ for adhesions, $22 \%$ for bowel perforations and $22 \%$ for technical difficulties with the instruments. The conversion rate is acceptable in our patients due to the nature of the disease. Mimica et $\mathrm{al}^{69}$ reported $16 \%$ conversion rate to laparotomy due to adhesions and one of these patients had perforation of the colon during the insertion of trocar.

We did not have any complication like wound site fluid leakage, wound sepsis or post procedure peritonitis. Krishnan et $\mathrm{al}^{71}$ reported $4 \%$ wound sites leakage, whereas Menzies et $\mathrm{al}^{66}$ reported $6 \%$ wound sites leakage, $3 \%$ bacterial peritonitis and $2 \%$ deaths from complication of laparoscopy. Nine patients died 
(11\%) in our series during the two months follow up period, none of them died due to the procedure related complication. All of these nine patients were HIV positive. The cause of deaths of five patients who died in the local hospital could not be established. Two of these five patients had appendicitis, and the most likely cause of death was HIV/ AIDS related disease.

\subsection{Laparotomy and Laparoscopy for TB abdomen:}

Laparotomy was performed in $74 \%$ cases in the 1980 's ${ }^{100}, 50 \%$ cases in the 1990 's ${ }^{101}$ and $26 \%$ cases in our series. Complications rate of laparotomy can be as high as $80 \%{ }^{100}$, and are associated with high mortality rates of $60 \% .{ }^{17}$ The mortality rate of laparotomy was $39 \%$ in our series.

The trend has been moving away from laparotomy towards minimally invasive procedures such as laparoscopic, endoscopic and percutaneous methods to obtain tissue samples for diagnosis. Laparotomy should be performed only when complications develop or diagnosis remains unclear inspite of these diagnostic modalities. ${ }^{102}$ There were numerous publications with interest in laparoscopy in the diagnosis of abdominal tuberculosis, but the biggest problems with these publications are: almost all of them are retrospective and small sample size, most of their patients had wet (ascitic) type of abdominal tuberculosis and none of the patients were HIV positive or not mentioned. ${ }^{21,70,71,72,73}$ In comparison most of our patients are HIV positive and almost of them had dry (plastic) type of tuberculosis. 


\subsection{Follow up:}

We were very disappointed with the level of follow up we achieved in our patients as we tried to ensure that they wound return to follow up and continue with the supervision of treatment. This is not unique to this study or the province in which it was conducted. Clarke et al ${ }^{17}$ reported only $7 \%$ of their patients with abdominal tuberculosis completed the 6-month review at a surgical clinic. In a 2005 report from Baragwanath Hospital in Soweto over an 8-week period, 1291 patients were diagnosed with tuberculosis. Of their cohort $74 \%$ had pulmonary tuberculosis, $80 \%$ of those tested for HIV were positive, $19 \%$ died in hospital and their follow up was poor, only half of the patients referred to tuberculosis clinic attended within two weeks (duration of drug supply from the hospital) of referral. ${ }^{103}$ Follow up at antiretroviral therapy (ART) clinic is also poor in South Africa. Dalal et al ${ }^{104}$ reported in 2008 from a Johannesburg ART clinic that $16 \%$ of their 1631 adult patients receiving ART discontinued follow up in a one year period. The reasons for abysmal follow up are multifactorial and require analysis and quality improvement strategies if we are to gain any benefit from improved diagnostics. 


\section{Limitations}

There were some limitations in our study. Our study population was mainly ethnic African and Crohn's disease was not a diagnostic issue due to its rarity in the Africans in comparison to the Indians and White populations in South Africa.

Laparoscopic specimens were not taken from every patient. Only the specimens with macroscopic features suggestive of tuberculosis were collected for investigations. We did design it to be relative to the situation in most nonacademic regional hospitals where the findings would then be applicable. It is unclear if extending the biopsies to a routine sampling of all accessible tissues and including molecular techniques in the analysis of these tissues would further enhance the sensitivity and specificity of laparoscopic sampling bank. We designed it as above but would we have got a better yield if we had biopsied even the "normal tissue".

It has to be recognized that the sensitivity and specificity analysis is based on a gold standard, which is less than ideal. This is because the sampling techniques are not a new tests, they are away of establishing the gold standard. However, as this was the composite gold standard based on the presence of any of the three criteria, we felt this type of analysis was justified.

There was no microbiological result from the tissue samples and only a few blood and fluid culture results. Specimens were divided into two parts; one for 
histological and the other for microbiological examinations. Due to the logistical constraints we were unable to retrieve the microbiological results back. Sampling of multiple pathological sites has a high yield and reveals alternate diagnosis and reduces the number in which a trial of therapy is necessary.

Our biggest limitation of the study is the loss of follow up of the patients. As I mentioned earlier due to the poor socio-economic factors we had very few patients who came back for a follow up visit. This negates the benefit of improving the diagnostic accuracy by laparoscopy and highlights the need to drastically improve treatment supervison. 


\section{Conclusions}

\section{General Statement:}

Diagnosis of abdominal tuberculosis was difficult in the 1960's and remains difficult currently because of its nonspecific clinical presentation, despite improvement in the technology and diagnostic tools. Due to the growing burden of disease, drug resistant disease, HIV co-infection and the complexities of integrated drug therapy it is very important to make a definitive early diagnosis and spare those without tuberculosis unnecessary and long term antituberculosis therapy with its potential side effects. The use of diagnostic aids and syndromic diagnosis of tuberculosis have largely been developed and validated in the context of pulmonary tuberculosis and their sensitivity in abdominal tuberculosis has not been adequately studied. The current patient profile in this series was completely different from those of many other series in the past, luminal gastrointestinal disease and clinical ascites were uncommon and most of our patients were HIV positive with abdominal lymphadenopathy. Hence luminal and fluid samples were not available by simple means to establish the diagnosis and leaving laparotomy with its attendant high morbidity and mortality or laparoscopy as the only way to take adequate tissue biopsies and make the definitive diagnosis. 


\section{Conclusions as related to specific study aims:}

1) The role of diagnostic laparoscopy:

This study has shown the feasibility of performing laparoscopy in the majority of patients with suspected tuberculosis. It has a low conversion and complication rate. It has a high yield to establish the diagnosis of abdominal tuberculosis (68\%) by sampling macroscopically pathological tissues. It also provides the alternative diagnosis in $11 \%$ of patients in this setting. Laparotomy should be reserved for patients presenting with acute complications; complete intestinal obstruction or peritonitis due to perforation. Rapid diagnosis may be further enhanced by utilizing fresh biopsy specimens for analysis by molecular techniques. The subgroup of patients with tuberculosis negative but non-specific chronic inflammation needs further evaluation before we can abandon a strategy of empiric therapy in these individuals. To avoid the morbidity and mortality of laparotomy, the misdiagnosis of other abdominal conditions, and unnecessary long term therapy, diagnostic laparoscopy and tissue sampling is a viable and reliable strategy in patients with suspected abdominal tuberculosis. 
2) Gross laparoscopic features of abdominal tuberculosis:

Laparoscopy was very useful to identify the abnormalities of viscera, mesentery and peritoneum in the form masses, enlarged lymph nodes, peritoneal nodules, omental cake and bowel adhesions, which were the common findings in this series.

3) Capability of laparoscopy to provide adequate specimens:

Laparoscopy was very useful to collect specimens from all the abnormal looking sites including a small amount of peritoneal fluid. Total 217 specimens were collected from different sites and 114 of them were positive for tuberculosis.

4) Clinical response of patients with abdominal tuberculosis:

Our aim was to evaluate the clinical response to appropriate therapy. Unfortunately the follow up of our patients was very poor. From the limited number of patients who attended the follow up clinic and who could be contacted telephonically, we found that the response to anti-TB therapy was good. 


\section{References}

1. Karim SSA, Churchyard GJ, Karim QA, Lawn SD. HIV infection and tuberculosis in South Africa: an urgent need to escalate the public health response. Lancet 2009; 374: 921-33

2. Global summary of the AIDS epidemic, WHO 2009. http://www.who.int/hiv/data/2009_global_summary.png

3. Department of Health, 2010. National Antenatal Sentinel HIV and Syphilis Prevalence Survey in South Africa, 2009. http://www.doh.gov.za/docs/reports-f.html

4. Alvarez GG, Thembela BL, Muller FJ, Clinch J, Singhal N, Cameron DW. Tuberculosis at Edendale hospital in Pietermaritzburg, KwaZulu Natal, South Africa. Int J Tuberc Lung Dis 2004; 8 (12): 1472-1478

5. Global health observatory: TB incidence. WHO 2009. http://www.who.int/gho/mdg/diseases/tuberculosis/situation_trends_incide nce/en/index.html

6. WHO Report 2003: Global Tuberculosis Control: Surveillance, Planning, Financing. http://whqlibdoc.who.int/hq/2003/WHO_CDS_TB_2003.316.pdf

7. Global health observatory data repository: Incidence of tuberculosis. WHO 2009. http://apps. who.int/ghodata/?vid=510

8. Wilson D, Nachega J, Morroni C, Chaisson R, Maartens G. Diagnosing smear-negative tuberculosis using case definitions and treatment response in HIV-infected adults. Int J Tuberc Lung Dis 2006; 10 (1): 31-38 
9. The Italian Cooperation in South Africa: Demographic and Health Indicators Kwa-Zulu Natal. Public document available online. http://www.italcoop.co.za/PublicDocuments/Demographic_Health_Statistic aldata_KW_EC_SA\%20_EN.pdf

10. Statistics South Africa. Mortality and causes of death in South Africa, 2008: findings from death notification. November 2010. http://www.statssa.gov.za/publications/P03093/P030932008.pdf

11. Cohen T, Wilson D, Murray M, Wallengren K, Alvarez GG, et al. The prevalence and drug sensitivity of tuberculosis among patients dying in hospital in KwaZulu-Natal, South Africa: A postmortem study. PLoS Medicine 2010; 7 (6): e1000296

12. Global health observatory: TB mortality. WHO 2009. http://www.who.int/gho/mdg/diseases/tuberculosis/situation_trends_mortali ty/en/index.html

13. Riquelme A, Calvo M, Salech F, Valderrama S, Pattilo A, et al. Value of adenosine deaminase (ADA) in ascitic fluid for the diagnosis of tuberculous peritonitis. J Clin Gastroenterol 2006; 40 (8): 705-710

14. Khan R, Abid S, Jafri W, Abbas Z, Hameed K, Ahmad Z. Diagnostic dilemma of abdominal tuberculosis in non-HIV patients: an ongoing challenge for physicians. World J Gastroenterol 2006; 12 (39): 6371-6375

15. Ghiya R, Naik E, Casanas B, Izurieta R, Marfatia Y. Clinicoepidemiological profile of HIV/TB coinfected patients in Vadodara, Gujarat. J Sex Transm Dis 2009; 30: 10-15 
16. Gilinsky NH, Marks IN, Kottler RE, Price SK. Abdominal tuberculosis: a 10-year review. S Afr Med J 1983; 64: 849-857

17. Clarke DL, Thomson SR, Bissety T, Madiba TE, Buccimazza I, Anderson F. A single surgical unit's experience with abdominal tuberculosis in the HIV/AIDS era. World J Surg 2007; 31 (5):1088-1097

18. Manohar A, Simjee AE, Haffejee AA, Pettengell KE. Symptoms and investigative findings in 145 patients with tuberculous peritonitis diagnosed by peritoneoscopy and biopsy over a five year period. Gut 1990; 31 (10): $1130-1132$

19. Menzies RI, Alsen H, Fitzgerald JM, Mohapeloea RG. Tuberculous peritonitis in Lesotho. Tubercle 1986; 67: 47-54

20. Cacala SR, Mafana E, Thomson SR, Smith A. Prevalence of HIV status and CD4 counts in a surgical cohort: their relationship to clinical outcome. Ann R Coll Surg Engl 2006; 88: 46-51

21. Al-Mulhim AA. Laparoscopic diagnosis of peritoneal tuberculosis. Surg Endosc 2004; 18: 1757-61

22. Singh B, Moodley J, Batitang S, Chetty R. Isolated pancreatic tuberculosis and obstructive jaundice, S Afr Med J 2002; 92 (5); 357-9

23. Desai CS, Joshi AG, Abraham P, Desai DC, Deshpande RB, et al. Hepatic tuberculosis in absence of disseminated abdominal tuberculosis. Ann Hepatol_2006; 5 (1): 41-3

24. Chow KM, Chow VC-Y, Szeto CC. Indications for peritoneal biopsy in tuberculous peritonitis. Am J Surg 2003; 185: 567-573 
25. Novis BH, Bank S, Marks IN. Gastrointestinal and peritoneal tuberculosis: a study of cases at Groote Schuur Hospital 1962-1971. S Afr Med J 1973; 47: $359-64$

26. Gunn A, Keddie NC. Abdominal tuberculosis. Br J Surg 1972; 59: 597-602

27. Uygur-Bayramicli O, Dabak G, Dabak R. A clinical dilemma: abdominal tuberculosis. World J Gastroenterol 2003; 9: 1098-1101

28. Talwani $\mathrm{R}$, Horvath JA. Tuberculous peritonitis in patients undergoing continuous ambulatory peritoneal dialysis: case report and review. Clin Infect Dis 2000; 31: 70-5

29. Chow KM, Chow VC, Hung LC, Wong SM, Szeto CC. Tuberculous peritonitis- associated mortality is high among patients waiting for the results of mycobacterial cultures of ascitic fluid samples. Clin Infect Dis 2002; 35 (4): 409-13

30. Tariq SM, Tariq S. Empirical treatment for Tuberculosis: Survey of Cases treated over 2 years in a London Area. JPMA 2004; 54:88

31. Pepys MB, Hirschfield GM. C-reactive protein: a critical update. J Clin Invest 2003; 111 (12): 1805-12

32. Choi C-M, Kang C-I, Jeung W-K, Kim D-H, Lee C-H, et al. Role of Creactive protein for the diagnosis of TB among military personal in South Korea. Int J Tuberc Lung Dis 2007; 11 (2): 233-236

33. Lawn SD, Wiktor S, Coulibaly D, Ackah AN, Lal RB. Serum C-reactive protein and detection of tuberculosis in persons co-infected with human immunodeficiency virus. Trans R Soc Trop Med Hyg 2001; 95: 41-41 
34. Sathar MA, Simjee AE, Coovadia YM, Soni PN, Moola SAH, et al. Ascitic fluid $\mathrm{Y}$ interferon concentrations and adenosine deaminase activity in tuberculous peritonitis. Gut 1995; 36: 419-421

35. Burgess LJ, Swanepoel CG, Taljaard JJF. The use of adenosine deaminase as a diagnostic tool for peritoneal tuberculosis. Tuberculosis $2001 ; 81(3): 243-8$

36. Hillebrand DJ, Runyon BA, Yasmineh WG, Rynders GP. Ascitic fluid adenosine deaminase insensitivity in detecting tuberculous peritonitis in the United States. Hepatology 1996; 24: 1408-12

37. Mas MR, Comert B, Saglamkaya U, Yamanel L, Kuzhan O, et al. CA-125; a new marker for diagnosis and follow-up of patients with tuberculous peritonitis. Digest Liver Dis 2000; 32: 595-7

38. Kulkarni S, Vyas S, Supe A, Kadival G. Use of polymerase chain reaction in the diagnosis of abdominal tuberculosis. J Gastroenterol Hepatol_2006; 21: $819-23$

39. Kahla IB, Selma WB, Marzouk M, Ferjeni A, Ghezal S, et al. Evaluation of a simplified IS6110 PCR for the rapid diagnosis of mycobacterium tuberculosis in an area with high tuberculosis incidence. Pathologie Biology 2009, doi: 10.1016/j.pathbio.2009.04.001

40. Kim S-H, Cho O-H, Park SJ, Ye BD, Sung H, et al. Diagnosis of abdominal tuberculosis by T-cell-based assay on peripheral blood and peritoneal fluid mononuclear cells. J Infect 2009; 59: 409-15 
41. Mazurek GH, Jereb J, LoBue $\mathrm{P}$, lademarco MF, Metchock B, et al. Guidelines for using the Quantiferon- TB gold test for detecting Mycobacterium tuberculosis infection, United States. MMWR: Recommendations and reports (CDC) 2005 Dec; 54 (RR15): 49-55

42. Agarwal D, Narayan S, Chakravarty J, Sundar S. Ultrasonography for diagnosis of abdominal tuberculosis in HIV infected people. Indian J Med Res 2010; 132: 77-80

43. Sharma MP, Bhatia V. Abdominal tuberculosis. Indian J Med Res 2004; 120: $305-315$

44. Sculier D, Vannarith C, Pe R, Thai S, Kanara N, et al. Performance of abdominal ultrasound for diagnosis of tuberculosis in HIV-infected persons living in Cambodia. J Acquir Immune Defic Syndr 2010; 55 (4): 500-502

45. Heller T, Goblirsch S, Wallrauch C, Lessells R, Brunetti E. Abdominal tuberculosis: sonographic diagnosis and treatment response in HIVpositive adults in rural South Africa. Int J Infect Dis 2010; 14S: e108-e112

46. Sinan T, Sheikh M, Ramadan S, Sahwney S, Behbehani A. CT features in abdominal tuberculosis: 20 years experience. BMC Medical Imaging 2002; $2(3)$

47. Pereira JM, Madureira AJ, Vieira A, Ramos I. Abdominal tuberculosis: imaging features. Eur J Radiol 2005; 55: 173-80

48. Ko CY, Schmit PJ, Petrie B, Thompson JE. Abdominal tuberculosis: the surgical perspective. Am Surg 1996; 62 (10): 865-8 
49. Yang Z, Sone S, Min P, Li F, Maruyama Y, et al. Distribution and contrast enhanced CT appearance of abdominal tuberculosis lymphadenopathy. Nippon lqaku Hoshasen Gakkai Zasshi 1997; 57 (9): 567-71

50. Niaz K, Ashraf M. Intestinal tuberculosis: diagnostic dilemma. Professional Med J 2010; 17 (4): 532-537

51. Epstein D, Watermeyer G, Kirsch R. The diagnosis and management of Crohn's disease in populations with high-risk rates for tuberculosis. Aliment Pharmacol Ther 2007; 25 (12): 1373-1388

52. Sato S, Yao K, Yao T, Schlemper RJ, Matsui T, et al. Colonoscopy in the diagnosis of intestinal tuberculosis in asymptomatic patients. Gastrointestinal Endoscopy 2004; 59 (3): 362-68

53. Chong VH, Lim KS. Gastrointestinal tuberculosis. Singapore Med J 2009; 50 (6): 638-646

54. Kumar R. Empirical use of anti-tuberculosis drugs should not be equated to their inappropriate and indiscriminate use. Indian J Pharm 2011; 43 (3): 363-364

55. Kumar R, Bhatia V, Khanal S, Sreenivas V, Gupta SD, et al. Antituberculosis therapy-induced acute liver failure: magnitude, profile, prognosis, and predictors of outcome. Hepatology 2010; 51 (5): 16651674

56. Chen H-L, Wu M-S, Chang W-H, Shih S-C, Chi H, et al. Abdominal tuberculosis in Southeastern Taiwan: 20 years of experience. J Formos Med Assoc 2009; 108 (3): 195-201 
57. Wolfe JH, Behn AR, Jackson BT. Tuberculous peritonitis and role of diagnostic laparoscopy. Lancet 1979; 1 (8121): 852-53

58. Yagmurlu A, Tolasa G, Yagmurlu B, Gokcora H, Dindar H. Abdominal tuberculosis mimicking malignancy: a case report. J Ank Med Sch 2002; $24(2): 91-94$

59. Sen M, Turan M, Karadayi K, Aslan M, Elagoz S. Peripancreatic tuberculous lymphadenitis mimicking carcinoma: report of a case. Acta Chir Belg 2004; 104: 338-340

60. Kapoor V. Abdominal tuberculosis. Medicine 2007; 35 (5): 257-260

61. Abbasi A, Javaherzadeh M, Arab M, Keshoofy M, Pojhan S, Daneshvar G. Surgical treatment for complications of abdominal tuberculosis. Arch Iranian Med 2004; 7 (1): 57-60

62. Donohue RF, Schnidor BI, Gorman J. Needle biopsy of the peritoneum. Arch Intern Med 1959; 103: 739-45

63. Cope C, Bernhardt H. Hook-needle biopsy of pleura, pericardium, peritoneum and synovium. Am J Med 1963; 35 (2): 189-95

64. Levine $\mathrm{H}$. Needle biopsy of peritoneum in exudative ascites. Arch Intern Med 1967; 120 (5): 542-45

65. Rodriguez de Lope C, San Miguel JG, Pons RF. Laparoscopic diagnosis of tuberculous ascites. Endoscopy 1982; 14 (5): 178-79

66. Menzies RI, Fitzgerald JM, Mulpeter K. Laparoscopic diagnosis of ascites in Lesotho. BMJ 1985; 291: 473-75 
67. Wang WN, Wallack MK, Barnhart S, Kalani AD, Storrs SL. Tuberculous peritonitis: definitive diagnosis by laparoscopic peritoneal biopsy. Am Surg 2008; 74 (12)1223-1224

68. Geake TMS, Spitaels JM, Moshal MG, Simjee AE. Peritoneoscopy in the diagnosis of tuberculous peritonitis. Gastrointestinal Endoscopy 1981; 27 (2): 66-68

69. Mimica M. Usefulness and limitations of laparoscopy in the diagnosis of tuberculous peritonitis. Endoscopy 1992; 24: 588-91

70. Mohamed AAR, Bhat NA, Abukhater M, Riaz MM. Role of laparoscopy in diagnosis of abdominal tuberculosis. The Internet Journal of Infectious Diseases 2010; Volume 8, Number 2

71. Krishnan P, Vayoth SO, Dhar P, Surendran S, Ponnambathayil S. Laparoscopy in suspected abdominal tuberculosis is useful as an early diagnostic method. ANZ J Surg 2008; 78: 987-989

72. Rai S, Thomas WM. Diagnosis of abdominal tuberculosis: the importance of laparoscopy. J R Soc Med 2003; 96: 586-8

73. Meshikhes A-W N. Pitfalls of diagnostic laparoscopy in abdominal tuberculosis. Surg Endosc 2010; 24:908-910

74. McLaughlin S, Jones T, Pitcher M, Evans P. Laparoscopic diagnosis of abdominal tuberculosis. Aust NZ J Surg 1998; 68: 599-601

75. Grau JMS, Chaves CR, Moreno JLG, Cartes JAM, Macias MS, et al. Atypical peritoneal tuberculosis, use of laparoscopy in the diagnosis. Rev Esp Enferm Dis 2007; 99 (12): 725-728 
76. Safarpor F, Aghajanzade M, Kohsari MRR, Hoda S, Sarshad A, et al. Role of laparoscopy in the diagnosis of abdominal tuberculosis. Saud J Gastroenterol 2007; 13 (3): 133-5

77. Orellana JM, Valdivia-Barriga V, Feldman A. Needle biopsy of the peritoneum. Dig Dis Sci 1964; 9 (2): 145-50

78. Nafeh MA, Madhat A, Abdul-Hameed AG, Ahmad YA, Rashwan NM, et al. Tuberculous peritonitis in Egypt: the value of laparoscopy in diagnosis. Am J Trop Med Hyg 1992; 47 (4): 470-7

79. Chou C-H, Ho M-W, Ho C-M, Lin P-C, Weng C-Y, et al. Abdominal tuberculosis in adult: 10-year experience in a teaching hospital in central Taiwan. J Microbiol Immunol Infect 2010; 43 (5): 395-400

80. Ramesh J, Banait GS, Ormerod LP. Abdominal tuberculosis in a district general hospital: a retrospective review of 86 cases. Q J Med 2008 (Jan); doi:10.1093/qjmed/hcm125

81. Sinkala E, Gray S, Zulu I, Mudenda V, Zimba L, et al. Clinical and ultrasonographic features of abdominal tuberculosis in HIV positive adults in Zambia. BMC Infectious Diseases 2009; 9:44. doi:10.1186/1471-2334$9-44$

82. lliyasu Z, Babashani M. Prevalence and predictors of tuberculosis coinfection among HIV-seropositive patients attending the Aminu Kano Teaching hospital, Northern Nigeria. J Epidemiol 2009; 19 (2): 81-87 
83. Kishore PV, Chandresekhar TS, Palaian S. Diagnosis of abdominal tuberculosis: a retrospective study from Nepal. The Internet Journal of Gastroenterology 2008; 6 (2)

84. Bolukbas C, Bolukbas FF, Kendir T, Dalay RA, Akbayir N, et al. Clinical presentation of abdominal tuberculosis in HIV seronegative adults. BMC Gastroenterology 2005; 5:21, doi:10.1186/1471-230x5-21

85. Levay PF, Viljoen M. Erythrocyte sedimentation rates equal to or above $100 \mathrm{~mm} / \mathrm{h}$ in the pre AIDS era. S Afr Med J 2002; 92 (4); 286-287

86. Levay PF, Retief JH. Causes of high erythrocyte sedimentation rates in an inpatient population. SAMJ 2005; 95 (1): 45-46

87. Ukpe IS, Southern L. Erythrocyte sedimentation rate values in active tuberculosis with and without HIV co-infection. SAMJ 2006; 96 (5); 427428

88. Al-Marri MRHA, Kirkpatrick MB. Erythrocyte sedimentation rate in childhood tuberculosis: is it still worthwhile? Int J Tuberc Lung DIs 2000; 4 (3): 237-239

89. Wilson D, Badri M, Maartens G. Performance of serum C-reactive protein as a screening test for smear-negative tuberculosis in an ambulatory high HIV prevalence population. PLoS ONE 2011; 6 (1): e15248

90. Sage EK, Noursadeghi M, Evans HE, Parker SJ, Copas AJ, et al. Prognostic value of C-reactive protein in HIV-infected patients with pneumocystis jirovecii pneumonia. Int J STD AIDS 2010; 21: 288-292 
91. Noursadeghi M, Miller RF. Clinical value of C-reactive protein measurements in HIV-positive patients. International Journal of STD \& AIDS 2005; 16: 438-441

92. Schleicher GK, Herbert V, Brink A, Martin S, Maraj R, et al. Procalcitonin and C-reactive protein levels in HIV-positive subjects with tuberculosis and pneumonia. Eur Respir J 2005; 25: 688-692

93. Chaudhary M, Kashyap B, Gautam H, Saini S, Bhalla P. Role of Creactive protein in HIV infection: a pilot study. Viral Immunology 2008; 21 (2): 263-266

94. Da Bouza J, Di Lonardo M, Benetucci J, Gonzalez MJ, Abbate E, et al. Blood culture value in the diagnostic of the disseminated tuberculosis in AIDS patients. International Conference in AIDS 1990; 6 (247): 20-23

95. Mishra PK, Bhargava A, Punde RP, Pathak N, Desikan P, et al. Diagnosis of gastrointestinal tuberculosis: using cytomorphological, microbiological, immunological and molecular techniques - a study from central India. Ind J Clin Biochem_2010; 25 (2): 158-163

96. Boehme CC, Nabeta P, Hillemann D, Nicol MP, Shenai S, et al. Rapid molecular detection of tuberculosis and rifampicin resistance. $\mathrm{N}$ Eng $\mathrm{J}$ Med 2010; 363 (11): 1005-1015

97. Evans CA. GeneXpert- a game-changer for tuberculosis control? PLoS Med 2011; 8 (7): e1001064

98. Marks IN. Abdominal tuberculosis. Bailliere's Clin Med Communicable Dis 1988; 3: 329-348 
99. Sotoudehmanesh R, Shirazian N, Asgari AA, Malekzadeh R. Tuberculous peritonitis in an endemic area. Digestive and Liver Diseases 2003; 35: $37-$ 40

100. Chen Y-M, Lee P-Y, Perng R-P. Abdominal tuberculosis in Taiwan: a report from Veterans' General Hospital, Taipei. Tubercle and Lung Disease 1995; 76: 35-38

101. Hassan I, Brilakis ES, Thomson RL, Que FG. Surgical management of abdominal tuberculosis. J Gastrointest Surg 2002; 6 (6): 862-867

102. Akgun Y. Intestinal and peritoneal tuberculosis: changing trends over 10 years and a review of 80 patients. Can J Surg 2005; 48 (2): 131-136

103. Edginton ME, Wong ML, Phofa R, MahlabaD, Hodkinson HJ. Tuberculosis at Chris Hani Baragwanath Hospital: numbers of patients diagnosed and outcomes of referrals to district clinics. Int $\mathrm{J}$ Tuberc Lung Dis 2005; 9 (4): $398-402$

104. Dalal RP, MacPhail C, Mqhayi M, Wing J, Feldman C, et al. Characteristics and outcomes of adult patients lost to follow up at an antiretroviral treatment clinic in Johannesburg, South Africa. J Acquir Immune Defic Syndr 2008; 47 (1): 101-7 


\section{Appendices}

Table 6: Breakdown of alternate diagnosis and TB positive patients in laparoscopic specimens.

\begin{tabular}{|c|c|c|c|c|c|c|c|c|}
\hline No. & Mass & Tubercle & $\begin{array}{l}\text { Lymph } \\
\text { nodes }\end{array}$ & Omentum & Peritoneum & $\begin{array}{l}\text { Ascitic } \\
\text { fluid }\end{array}$ & Liver & $\begin{array}{l}\text { Other } \\
\text { diagnosis }\end{array}$ \\
\hline \multicolumn{9}{|l|}{1.} \\
\hline 2. & + & & + & & & & & \\
\hline \multicolumn{9}{|l|}{3.} \\
\hline 4. & & & + & & & & & \\
\hline 5. & & & & & & & & Appendicitis \\
\hline 6. & & + & & + & + & & & \\
\hline 7. & & & & + & & & & \\
\hline \multicolumn{9}{|l|}{8.} \\
\hline 9. & + & & + & + & & & & \\
\hline \multicolumn{9}{|l|}{10.} \\
\hline 11. & + & & + & & & & & \\
\hline 12. & + & & + & & & + & & \\
\hline 13. & + & + & + & & & + & & \\
\hline 14. & & & & & & + & & \\
\hline \multicolumn{9}{|l|}{15.} \\
\hline 16. & & + & & & & & & \\
\hline 17. & + & + & + & + & & & & \\
\hline 18. & & & + & & & & & \\
\hline 19. & & & + & & & & & \\
\hline 20. & + & & + & & & & & \\
\hline
\end{tabular}




\begin{tabular}{|c|c|c|c|c|c|c|c|}
\hline 21. & & & & & & & \\
\hline 22. & + & & + & & & & \\
\hline 23. & + & + & + & + & + & & \\
\hline 24. & & & & & & & \\
\hline 25. & & & & & & & \\
\hline 26. & & & + & & & & \\
\hline 27. & + & + & + & & & & \\
\hline 28. & & & & & & & Appendicitis \\
\hline 29. & & & + & & + & & \\
\hline 30. & & & & & + & + & \\
\hline 31. & & + & + & & & & \\
\hline 32. & & & + & & & + & \\
\hline 33. & & + & + & + & & + & \\
\hline 34. & & + & + & & & + & \\
\hline 35. & + & & + & & & & \\
\hline 36. & & & + & & & & \\
\hline 37. & & & + & & & & \\
\hline 38. & & + & & + & + & & \\
\hline 39. & & & & & & & \\
\hline 40. & & & & & & & Portal HPT \\
\hline 41. & & & + & & & & \\
\hline 42. & & & & & & & Adeno Ca \\
\hline 43. & + & & + & & & & \\
\hline 44. & & & + & & & & \\
\hline 45. & & + & & + & & & \\
\hline 46. & & & & & & & Adeno $\mathrm{Ca}$ \\
\hline 47. & & & + & & & & \\
\hline
\end{tabular}




\begin{tabular}{|c|c|c|c|c|c|c|c|c|}
\hline 48. & & & & & & & & \\
\hline 49. & & & & & & + & & \\
\hline 50. & & + & + & & & & & \\
\hline 51. & & & + & & & + & & \\
\hline 52. & & & & & & & & \\
\hline 53. & & & & & & & & Perforation \\
\hline 54. & & & + & & & + & & \\
\hline 55. & & & & & + & & & \\
\hline 56. & + & & + & & + & & & \\
\hline 57. & & & + & & & & & \\
\hline 58. & & & + & & & & & \\
\hline 59. & & + & + & + & + & & & \\
\hline 60. & & & + & + & & & & \\
\hline 61. & & & & & & & & \\
\hline 62. & & & & & & & & \\
\hline 63. & & + & + & & & & & \\
\hline 64. & & & + & & & + & & \\
\hline 65. & & & + & & & & & \\
\hline 66. & & + & & + & & & & \\
\hline 67. & & & & & & + & & \\
\hline 68. & + & & + & + & & + & & \\
\hline 69. & & & & & & & & Adeno $\mathrm{Ca}$ \\
\hline 70. & & & & & & & & Appendicitis \\
\hline 71. & & & & & & & & \\
\hline 72. & & & & & & & & \\
\hline 73. & & & + & & & + & + & \\
\hline 74. & & & & + & & & & \\
\hline
\end{tabular}




\begin{tabular}{|c|c|c|c|c|c|c|c|c|}
\hline 75. & & & & & & & & Lymphoma \\
\hline 76. & & & + & + & + & + & & \\
\hline 77. & & & & & & & & No specimen \\
\hline 78. & & & & & & & & \\
\hline 79. & & & + & & & & & \\
\hline 80. & & & + & & & + & & \\
\hline 81. & & + & + & + & & & & \\
\hline $\begin{array}{l}\text { Total } \\
\text { positive }\end{array}$ & 14 & 16 & 43 & 15 & 9 & 16 & 1 & 10 \\
\hline $\begin{array}{l}\text { Grand } \\
\text { Total }\end{array}$ & \multicolumn{7}{|c|}{114 (Tuberculosis) } & 10 \\
\hline
\end{tabular}


Table 9: Non- specific chronic inflammation / no pathology in 16 patients.

\begin{tabular}{|c|c|c|c|}
\hline Case no. & $\begin{array}{l}\text { TB negative and } \\
\text { No other diagnosis }\end{array}$ & $\begin{array}{l}\text { Non specific } \\
\text { chronic inflammation }\end{array}$ & No pathology \\
\hline 1. & 1 & & + \\
\hline \multicolumn{4}{|l|}{2.} \\
\hline 3. & 2 & + & \\
\hline \multicolumn{4}{|l|}{4.} \\
\hline \multicolumn{4}{|l|}{5.} \\
\hline \multicolumn{4}{|l|}{6.} \\
\hline \multicolumn{4}{|l|}{7.} \\
\hline 8. & 3 & + & \\
\hline \multicolumn{4}{|l|}{9.} \\
\hline 10. & 4 & + & \\
\hline 11. & & + & \\
\hline \multicolumn{4}{|l|}{12.} \\
\hline \multicolumn{4}{|l|}{13.} \\
\hline 14. & & & + \\
\hline 15. & 5 & + & \\
\hline \multicolumn{4}{|l|}{16.} \\
\hline \multicolumn{4}{|l|}{17.} \\
\hline \multicolumn{4}{|l|}{18.} \\
\hline \multicolumn{4}{|l|}{19.} \\
\hline \multicolumn{4}{|l|}{20.} \\
\hline 21. & 6 & + & \\
\hline \multicolumn{4}{|l|}{22.} \\
\hline 23. & & & \\
\hline
\end{tabular}




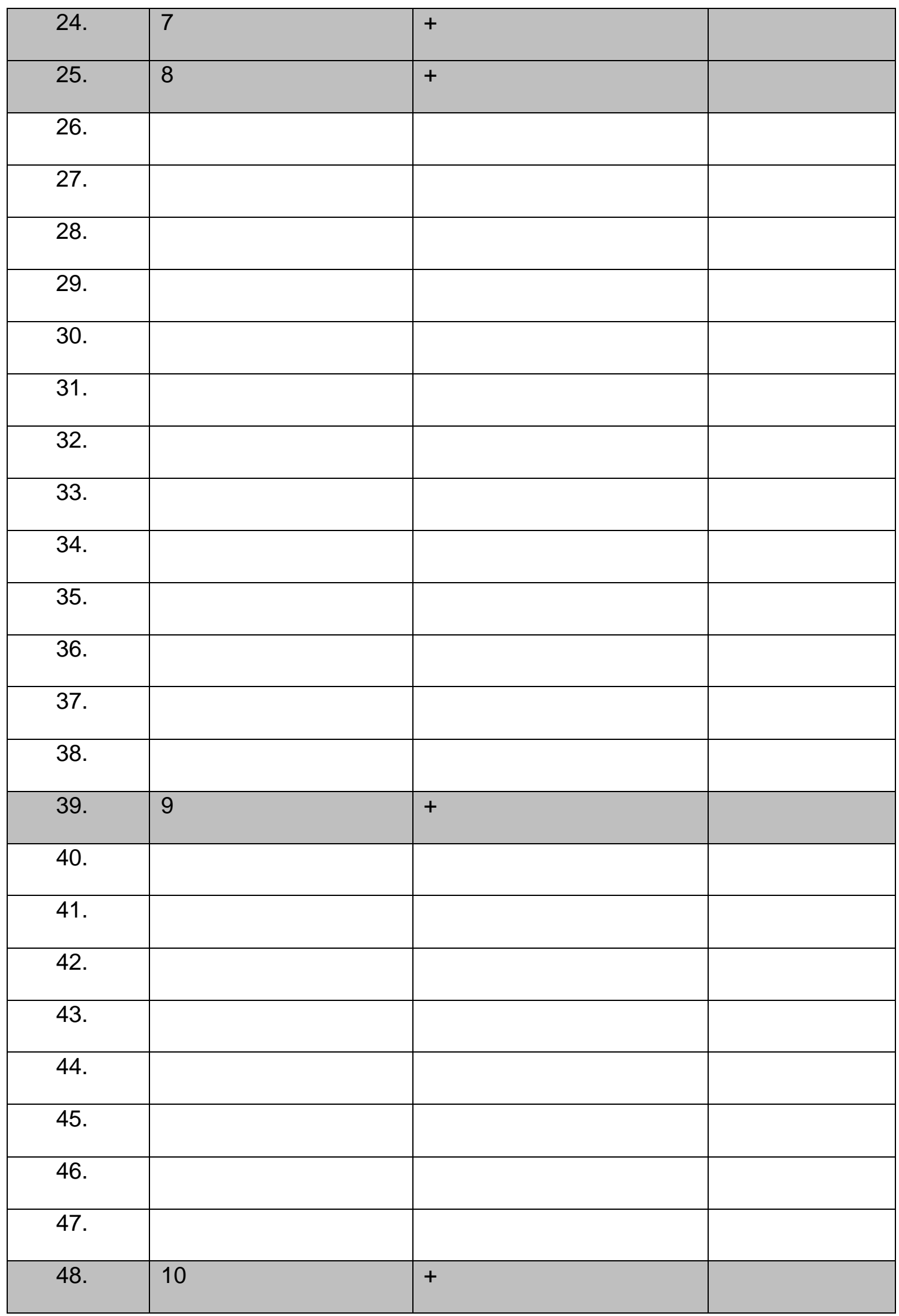




\begin{tabular}{|c|c|c|c|}
\hline 49. & & + & \\
\hline \multicolumn{4}{|l|}{50 . } \\
\hline 51. & & + & + \\
\hline 52. & 11 & + & + \\
\hline \multicolumn{4}{|l|}{53.} \\
\hline 54. & & & + \\
\hline \multicolumn{4}{|l|}{55.} \\
\hline 56. & & & + \\
\hline 57. & & + & + \\
\hline 58. & & & + \\
\hline \multicolumn{4}{|l|}{59.} \\
\hline 60. & & & + \\
\hline 61. & 12 & + & + \\
\hline 62. & 13 & + & \\
\hline \multicolumn{4}{|l|}{63.} \\
\hline \multicolumn{4}{|l|}{64.} \\
\hline \multicolumn{4}{|l|}{65.} \\
\hline \multicolumn{4}{|l|}{66.} \\
\hline 67. & & & + \\
\hline \multicolumn{4}{|l|}{68.} \\
\hline \multicolumn{4}{|l|}{69.} \\
\hline 70. & & + & \\
\hline 71. & 14 & + & \\
\hline 72. & 15 & + & + \\
\hline 73. & & & \\
\hline
\end{tabular}




\begin{tabular}{|c|l|l|l|}
\hline 74. & & & + \\
\hline 75. & & & \\
\hline 76. & & & + \\
\hline 77. & & & \\
\hline 78. & 16 & + & \\
\hline 79. & & & \\
\hline 80. & & & + \\
\hline 81. & & & \\
\hline Total & 16 & 20 & 15 \\
\hline
\end{tabular}


Table 10: Breakdown of histology/ blood culture/ ascitic fluid culture results.

\begin{tabular}{|c|c|c|c|c|}
\hline \begin{tabular}{|l} 
Case No. \\
.
\end{tabular} & $\begin{array}{l}\text { Histology } \\
\text { TB positive }\end{array}$ & $\begin{array}{l}\text { Blood culture } \\
\text { TB positive }\end{array}$ & $\begin{array}{l}\text { Ascitic fluid culture } \\
\text { TB positive }\end{array}$ & $\begin{array}{l}\text { Other } \\
\text { diagnosis }\end{array}$ \\
\hline \multicolumn{5}{|c|}{ - posion a } \\
\hline 2 & + & & & \\
\hline \multicolumn{5}{|l|}{3} \\
\hline 4 & + & & & \\
\hline 5 & & & & + \\
\hline 6 & + & & & \\
\hline 7 & + & & & \\
\hline \multicolumn{5}{|l|}{8} \\
\hline 9 & + & & & \\
\hline \multicolumn{5}{|l|}{10} \\
\hline 11 & + & & & \\
\hline 12 & + & + & + & \\
\hline 13 & + & + & + & \\
\hline 14 & & & $\begin{array}{lll}+ & & \\
\end{array}$ & \\
\hline \multicolumn{5}{|l|}{15} \\
\hline 16 & + & & & \\
\hline 17 & + & + & 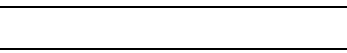 & \\
\hline 18 & + & & & \\
\hline 19 & + & & & \\
\hline \multirow{2}{*}{\multicolumn{5}{|c|}{21}} \\
\hline & & & & \\
\hline 22 & + & + & & \\
\hline 23 & + & & & \\
\hline \multicolumn{5}{|l|}{24} \\
\hline \multicolumn{5}{|l|}{25} \\
\hline 26 & + & & & \\
\hline 27 & + & & & \\
\hline 28 & & & & + \\
\hline 29 & + & & & \\
\hline 30 & + & + & + & \\
\hline 31 & + & & & \\
\hline 32 & + & + & $\begin{array}{lll}+ & & \\
\end{array}$ & \\
\hline 33 & + & & + & \\
\hline 34 & + & + & + & \\
\hline 35 & + & + & & \\
\hline 36 & + & & & \\
\hline 37 & + & & & \\
\hline 38 & + & & & \\
\hline \multicolumn{5}{|l|}{39} \\
\hline 40 & & & & + \\
\hline 41 & + & & & \\
\hline 42 & & & & + \\
\hline 43 & + & & & \\
\hline 44 & + & + & & \\
\hline 45 & + & + & & \\
\hline 46 & & & & + \\
\hline 47 & + & & & \\
\hline
\end{tabular}




\begin{tabular}{|c|c|c|c|c|}
\hline \multicolumn{5}{|l|}{48} \\
\hline 49 & & + & + & \\
\hline 50 & + & & & \\
\hline 51 & + & + & + & \\
\hline \multicolumn{5}{|l|}{52} \\
\hline 53 & & & & + \\
\hline 54 & + & & + & \\
\hline 55 & + & & & \\
\hline 56 & + & & & \\
\hline 57 & + & & & \\
\hline 58 & + & & & \\
\hline 59 & + & & & \\
\hline 60 & + & & & \\
\hline \multicolumn{5}{|l|}{61} \\
\hline \multicolumn{5}{|l|}{62} \\
\hline 63 & + & & & \\
\hline 64 & + & & + & \\
\hline 65 & + & & & \\
\hline 66 & + & & & \\
\hline 67 & & + & + & \\
\hline 68 & + & & + & \\
\hline 69 & & & & + \\
\hline 70 & & & & + \\
\hline \multicolumn{5}{|l|}{71} \\
\hline \multicolumn{5}{|l|}{72} \\
\hline 73 & + & & + & \\
\hline 74 & + & & & \\
\hline 75 & & & & + \\
\hline 76 & + & & + & \\
\hline 77 & & & & No specimen \\
\hline \multicolumn{5}{|l|}{78} \\
\hline 79 & + & & & \\
\hline 89 & + & & + & \\
\hline 81 & + & & & \\
\hline $\begin{array}{l}\text { Total } \\
\text { positive }\end{array}$ & 52 & 13 & 16 & 10 \\
\hline
\end{tabular}


Table 11: Breakdown of 26 patients who were negative for TB.

\begin{tabular}{|c|c|c|c|c|c|c|c|c|c|}
\hline No. & Age & Sex & HIV status & Other diagnosis & $\begin{array}{l}\text { Non-specific } \\
\text { chronic } \\
\text { inflammation }\end{array}$ & $\begin{array}{l}\text { No } \\
\text { pathology }\end{array}$ & ESR & CRP & CT scan \\
\hline 1 & 23 & $\mathrm{~F}$ & Negative & --- & --- & + & 75 & --- & + \\
\hline 2 & 23 & $\mathrm{~F}$ & Negative & $\begin{array}{ll}-- \\
-\end{array}$ & + & & $\begin{array}{ll}-- \\
-\end{array}$ & --- & + \\
\hline 4 & 42 & $\mathrm{M}$ & Positive & --- & + & & 45 & 5 & + \\
\hline 5 & 51 & $M$ & Positive & --- & + & & 90 & --- & --- \\
\hline 6 & 28 & $\mathrm{~F}$ & Positive & --- & + & & --- & --- & + \\
\hline 7 & 21 & $\mathrm{~F}$ & Positive & --- & + & & --- & --- & --- \\
\hline 8 & 30 & $\mathrm{~F}$ & Positive & --- & + & & --- & --- & --- \\
\hline 9 & 49 & $\mathrm{~F}$ & Negative & --- & + & & 13 & 96 & + \\
\hline 10 & 61 & $\mathrm{M}$ & Unknown & Appendicitis & --- & & --- & --- & --- \\
\hline 11 & 25 & $\mathrm{M}$ & Positive & $\begin{array}{ll}-- \\
\end{array}$ & + & & $\begin{array}{ll}--- \\
\end{array}$ & $\begin{array}{ll}-- \\
\end{array}$ & + \\
\hline 12 & 16 & $\mathrm{~F}$ & Unknown & Portal hypertension & $\begin{array}{ll}--- \\
\end{array}$ & & $\begin{array}{ll}--- \\
\end{array}$ & $\begin{array}{ll}-- \\
-1\end{array}$ & + \\
\hline 13 & 20 & $\mathrm{M}$ & Negative & Adeno carcinoma & --- & & --- & --- & + \\
\hline 14 & 25 & $\mathrm{M}$ & Positive & Adeno carcinoma & --- & & $\begin{array}{ll}--- \\
\end{array}$ & $\begin{array}{ll}-- \\
-1\end{array}$ & + \\
\hline
\end{tabular}




\begin{tabular}{|c|c|c|c|c|c|c|c|c|c|}
\hline 15 & 35 & $\mathrm{M}$ & Positive & --- & + & & --- & --- & + \\
\hline 16 & 33 & $\mathrm{~F}$ & Positive & --- & + & & --- & --- & + \\
\hline 17 & 18 & $M$ & Positive & $\begin{array}{l}\text { Non-specific bowel } \\
\text { perforation }\end{array}$ & $\begin{array}{ll}--- \\
\end{array}$ & & --- & --- & + \\
\hline 18 & 17 & $M$ & Positive & --- & + & & --- & --- & --- \\
\hline 19 & 47 & $\mathrm{~F}$ & Positive & --- & + & & --- & --- & + \\
\hline 20 & 35 & $\mathrm{~F}$ & Positive & Adeno carcinoma & --- & & 88 & 128 & --- \\
\hline 21 & 35 & $\mathrm{~F}$ & Positive & Appendicitis & --- & & 130 & 61 & + \\
\hline 22 & 17 & $M$ & Unknown & --- & + & & 60 & 48 & + \\
\hline 24 & 46 & $\mathrm{M}$ & Positive & Lymphoma & --- & & 78 & 96 & --- \\
\hline 25 & 31 & $\mathrm{M}$ & Positive & No specimen taken & $\begin{array}{ll}-- \\
\end{array}$ & & 105 & 24 & --- \\
\hline 26 & 28 & $\mathrm{~F}$ & Positive & --- & + & & 46 & 48 & --- \\
\hline Total & & & $\begin{array}{l}\text { Positive: } 18 \\
\text { Unknown: } 3 \\
\text { Negative: } 5\end{array}$ & $\begin{array}{l}\text { Other diagnosis: } 9 \\
\text { No specimen: } 1\end{array}$ & 15 & 1 & $\begin{array}{l}\text { High: } \\
10\end{array}$ & High: 8 & Suggestive: 17 \\
\hline
\end{tabular}


Table 12: Breakdown of 16 patients with TB negative and no other diagnosis.

\begin{tabular}{|c|c|c|c|c|c|c|c|c|}
\hline No. & Age & Sex & HIV status & $\begin{array}{l}\text { Non specific } \\
\text { chronic } \\
\text { inflammation }\end{array}$ & $\begin{array}{l}\text { No } \\
\text { pathology }\end{array}$ & ESR & CRP & CT Scan \\
\hline 1 & 23 & $\mathrm{~F}$ & Negative & --- & + & 75 & --- & + \\
\hline 2 & 23 & $\mathrm{~F}$ & Negative & + & --- & --- & \begin{tabular}{|l}
-- \\
\end{tabular} & + \\
\hline 3 & 42 & $M$ & Positive & + & --- & 45 & 5 & + \\
\hline 4 & 51 & $M$ & Positive & + & --- & 90 & --- & --- \\
\hline 5 & 28 & $\mathrm{~F}$ & Positive & + & --- & --- & --- & + \\
\hline 6 & 21 & $\mathrm{~F}$ & Positive & + & --- & --- & --- & --- \\
\hline 7 & 30 & $\mathrm{~F}$ & Positive & + & --- & --- & --- & --- \\
\hline 8 & 49 & $\mathrm{~F}$ & Negative & + & --- & 13 & 96 & + \\
\hline 9 & 25 & $M$ & Positive & + & --- & --- & --- & + \\
\hline 10 & 35 & $M$ & Positive & + & --- & --- & --- & + \\
\hline 11 & 33 & $\mathrm{~F}$ & Positive & + & --- & --- & --- & + \\
\hline 12 & 17 & $M$ & Positive & + & --- & --- & --- & --- \\
\hline 13 & 47 & $\mathrm{~F}$ & Positive & + & --- & --- & --- & + \\
\hline 14 & 17 & $\mathrm{M}$ & Unknown & + & --- & 60 & 48 & + \\
\hline 15 & 19 & $\mathrm{~F}$ & Negative & + & --- & 19 & 12 & + \\
\hline 16 & 28 & $\mathrm{~F}$ & Positive & + & --- & 46 & 48 & --- \\
\hline Total & & & $\begin{array}{l}11 \mathrm{HIV}+ \\
1 \text { unknown } \\
4 \text { negative }\end{array}$ & 15 & 1 & High: 5 & High: 4 & Suggestive: 11 \\
\hline
\end{tabular}




\section{List of tables}

Page

Table 1: Comparison of the frequency of the various diagnostic criteria

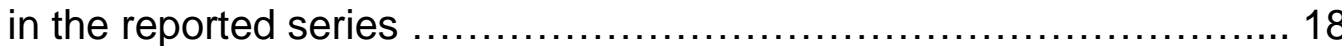

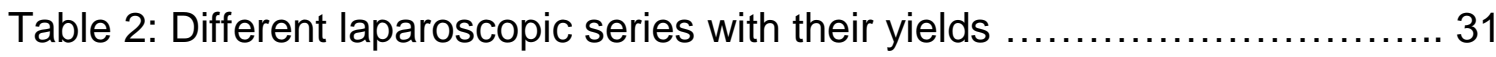

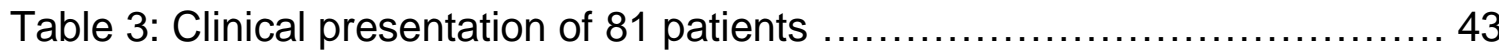

Table 4: Diagnostic yields of pre-operative investigations .................... 51

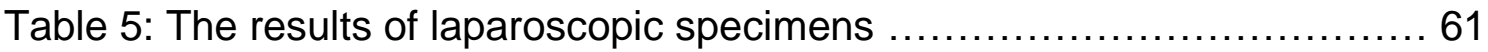

Table 6: Breakdown of alternate diagnosis and TB positive patients in

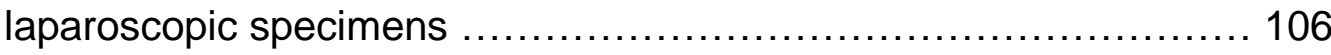

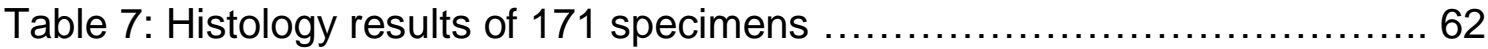

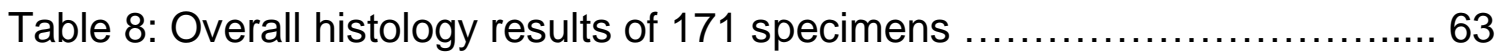

Table 9: Non- specific chronic inflammation / no pathology

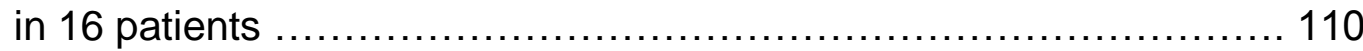

Table 10: Breakdown of histology/ blood culture/ ascitic fluid

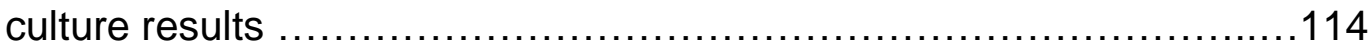

Table 11: Breakdown of 26 patients who were negative for TB ...............116

Table 12: Breakdown of 16 patients with TB negative and no other diagnosis ..................................................... 118

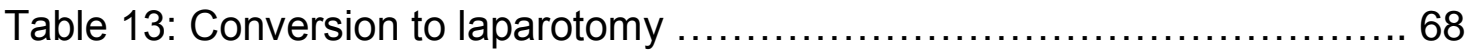




\section{List of figures}

Page

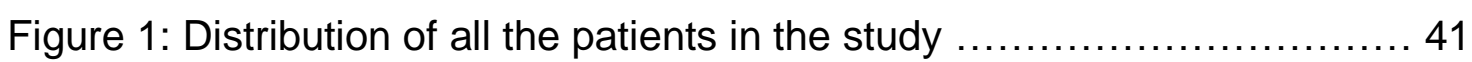

Figure 2: CT scan showing enlarged retroperitoneal lymph nodes (arrow)

with central area of necrosis; histology was positive for TB 46

Figure 3: CT scan showing enlarged retroperitoneal lymph nodes (arrow),

histology was negative for TB

Figure 4: CT scan showing enlarged retroperitoneal lymph nodes (arrow),

but no gland could be biopsied

Figure 5: CT scan showing enlarged mesenteric lymph nodes (thin arrow) and thickened mesentery (bold arrow), histology showed metastatic adenocarcinoma

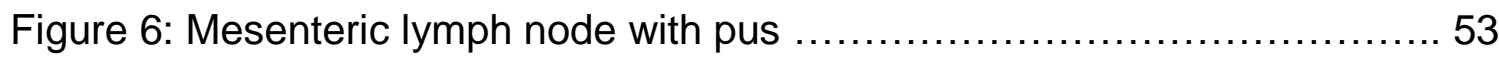

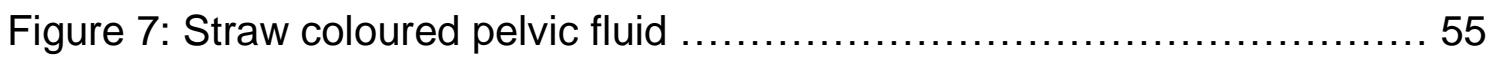

Figure 8: Taking biopsy from the omentum ............................... 56

Figure 9: Multiple tubercles over the lleo-caecal region ....................... 58

Figure 10: Tuberculosis positive result according to the number of

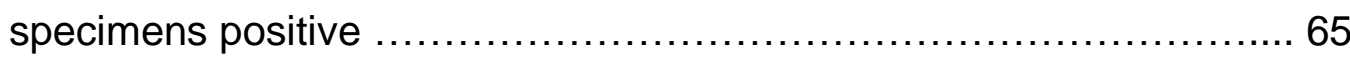

Figure 11: Laparoscopic results in relation to HIV status .......................6 67

Figure 12: Follow up of 81 patients ........................................... 70 


\section{List of abbreviations}

HIV : Human Immunodeficiency Virus

AIDS : Acquired Immune Deficiency Syndrome

TB : Tuberculosis

AFB : Acid-Fast Bacilli

ESR : Erythrocyte Sedimentation Rate

CRP : C - reactive protein

ADA : Adenosine Deaminase

CD4 : Cluster differentiation 4

CA-125 : Carcinogenic antigen-125

PCR : Polymerized Chain Reaction

RD-1 : Region of difference-1

ELISA : Enzyme Linked Immunosorbent Assay

IFN-y : Interferon Gamma

CXR : Chest radiograph

U/S : Ultrasound

CT : Computerized tomography

MDR : Multi drug resistant

BREC : Biomedical Research Ethics Committee

$\mathrm{Hb}$ : Haemoglobin

$\mathrm{Cl}$ : Confidence interval

MS : Microsoft 

WCC : White cell count
Gl : Gastrointestinal
PPV : Positive Predictive Value
NPV : Negative Predictive Value
$\mathrm{INH} \quad$ : Isoniazide 